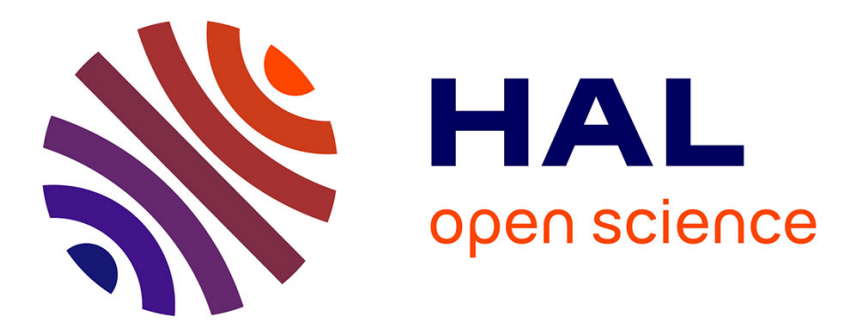

\title{
Towards nanoparticles with site-specific degradability by ring-opening copolymerization induced self-assembly in organic medium
}

Chen Zhu, Julien Nicolas

\section{- To cite this version:}

Chen Zhu, Julien Nicolas. Towards nanoparticles with site-specific degradability by ring-opening copolymerization induced self-assembly in organic medium. Polymer Chemistry, 2021, 12 (4), pp.594607. 10.1039/D0PY01425G . hal-03288977

\section{HAL Id: hal-03288977 \\ https://hal.science/hal-03288977}

Submitted on 16 Jul 2021

HAL is a multi-disciplinary open access archive for the deposit and dissemination of scientific research documents, whether they are published or not. The documents may come from teaching and research institutions in France or abroad, or from public or private research centers.
L'archive ouverte pluridisciplinaire HAL, est destinée au dépôt et à la diffusion de documents scientifiques de niveau recherche, publiés ou non, émanant des établissements d'enseignement et de recherche français ou étrangers, des laboratoires publics ou privés. 


\section{Towards Nanoparticles with Site-Specific}

\section{Degradability by Ring-Opening Copolymerization Induced Self-Assembly in Organic Medium}

Chen Zhu' ${ }^{1}$, Julien Nicolas*

${ }^{1}$ Université Paris-Saclay, CNRS, Institut Galien Paris-Saclay, 92296 Châtenay-Malabry,

France

*To whom correspondence should be addressed.

Email: julien.nicolas@u-psud.fr

Tel.: +33146835853 


\begin{abstract}
Reversible addition-fragmentation chain transfer (RAFT)-mediated radical ring-opening copolymerization-induced self-assembly (rROPISA) in heptane at 15 wt.\% solids was successfully applied to the copolymerization of benzyl methacrylate (BzMA) with 2methylene-1,3-dioxepane (MDO) from a poly(lauryl methacrylate) (PLMA) macro-RAFT agent. Despite a less efficient control of the copolymerization compared to similar copolymerizations with 2-methylene-4-phenyl-1,3-dioxolane (MPDL) or 5,6-benzo-2methylene-1,3-dioxepane (BMDO), it yielded stable, diblock copolymer nanospheres $\left(D_{\mathrm{z}}=\right.$ 100-200 nm) containing tunable amounts of polycaprolactone (PCL)-like degradable units in the core $\left(F_{\mathrm{MDO}}=0.04-0.19\right)$, resulting in significant degradation under accelerated hydrolytic conditions. Synthesis of surface-degradable diblock copolymer nanoparticles was then performed through the synthesis of $\mathrm{P}(\mathrm{LMA}-\mathrm{co}-\mathrm{BMDO})$ and $\mathrm{P}(\mathrm{LMA}-\mathrm{co}-\mathrm{MPDL})$ macro-chain transfer agents (CTAs). Even though partial livingness during chain extension with BzMA by PISA was noticed, stable nanospheres were obtained for $F_{\mathrm{BMDO}}=0.05$ and porous nanospheres for $F_{\mathrm{BMDO}}=0.08-0.09$, whose average diameters increased with the BMDO content. Attempts to prepare "all-degradable" vinyl particles were also made by rROPISA of BzMA and BMDO (or MPDL) from degradable macro-CTAs showing degradation in agreement with the copolymers' expected structure.
\end{abstract}




\section{Introduction}

Polymerization-induced self-assembly $(\mathrm{PISA})^{1-6}$ readily enables the one pot preparation of well-defined block copolymer nano-objects exhibiting different morphologies (e.g., spheres, ${ }^{7,8}$ worms $^{9,10}$ or vesicles ${ }^{11-15}$ ) at high concentrations and without any additional surfactant. These features represent important advantages in comparison to traditional polymerization processes in dispersed media (e.g., emulsion, dispersion). ${ }^{16}$ From a mechanistic point of view, dispersion PISA is based on the chain-extension of a solvophilic living polymer, previously synthesized by reversible deactivation radical polymerization (RDRP), by polymerization of a solvophilic monomer whose polymer becomes solvophobic at a certain chain length, leading to in situ selfassembly of the resultant amphiphilic diblock copolymers. PISA also exhibits additional benefits such as its applicability to different controlled polymerization techniques ${ }^{17-21}$ and its tolerance to a broad range of different reaction conditions ${ }^{1,3,5,22}$ and monomer families. ${ }^{19,22,23}$ In addition to receiving tremendous fundamental interest, the PISA technique has also shown promising results in the fields of materials science ${ }^{24}$, catalysis ${ }^{25}$ and biomedical applications. ${ }^{26-}$

Yet, one important application area in which polymerization processes in dispersed media (including PISA) have received very little attention is the synthesis of degradable particles. ${ }^{30-33}$ Indeed, polymer particles obtained by polymerization of traditional vinyl monomers are not degradable, which may greatly contribute to waste disposal problems and environmental issues. Therefore, new synthetic strategies for the development of degradable and/or environmentally friendly vinyl polymeric materials are highly desirable. ${ }^{34}$ 
In this context, our group recently developed radical ring-opening polymerization-induced self-assembly (rROPISA) ${ }^{31}$ using cyclic ketene acetals (CKA) to yield core-degradable nanoparticles in non-polar solvent. Radical ring-opening polymerization (rROP) of CKA is indeed one of the most efficient methods to confer degradability to vinyl polymers via incorporation of multiple ester groups in the polymer backbone. ${ }^{35,36}$ It has recently received increasing attention thanks to the ability of CKA to copolymerize with some traditional vinyl monomers by both free-radical polymerization and RDRP. ${ }^{37}$ In the past few years, rROP of CKAs has made significant progress from both fundamental ${ }^{38,39}$ and applicative ${ }^{40-43}$ perspectives. ${ }^{44}$ The rROPISA process was illustrated by the copolymerization in heptane between benzyl methacrylate (BzMA) and two different CKAs, either 2-methylene-4-phenyl1,3-dioxolane (MPDL) or 5,6-benzo-2-methylene-1,3-dioxepane (BMDO), from a poly(lauryl methacrylate) (PLMA) macro-chain transfer agents (CTAs). ${ }^{31}$ It led to stable diblock copolymer nanoparticles with narrow particle size distribution and tunable amounts of ester groups in the core block, and thus adjustable degradation rates of the copolymers under accelerated hydrolytic degradation conditions.

Although the rROPISA concept was validated in terms of CKA incorporation, selfassembly into narrowly dispersed particles and copolymer degradation, it raised the following important questions: (i) MPDL and BMDO were chosen for their structural similarity with BzMA but one could wonder whether rROPISA is also compatible with the more popular 2methylene-1,3-dioxepane (MDO), that leads to biodegradable polycaprolactone (PCL)-like units once opened. ${ }^{35}$ Indeed, incorporation of aromatic groups from MPDL and BMDO into the copolymer significantly impacts its physical properties and does not provide a satisfying route 
towards PCL-like polymers; (ii) Also, while vesicles and worms were expected at the block length ratios tested, only spherical nanoparticles were obtained, which highlighted the detrimental influence of MPDL and BMDO on the morphology. Would MDO lead to same observation? (iii) Eventually, it would be crucial to see whether rROPISA is versatile enough to allow incorporation of CKA not only into the solvophilic block to yield surface-degradable particles, but also in both blocks to induce degradation of the whole diblock copolymer.

Herein, we propose to provide first answers to those questions by investigating: (i) rROPISA with MDO as a CKA (Figure 1a) and (ii) rROPISA from CKA-containing macroCTA to yield surface-degradable (Figure 1b) and surface- plus core-degradable particles (Figure 1c) as a proof of concept of vinyl nanoparticles with site-specific degradability. 


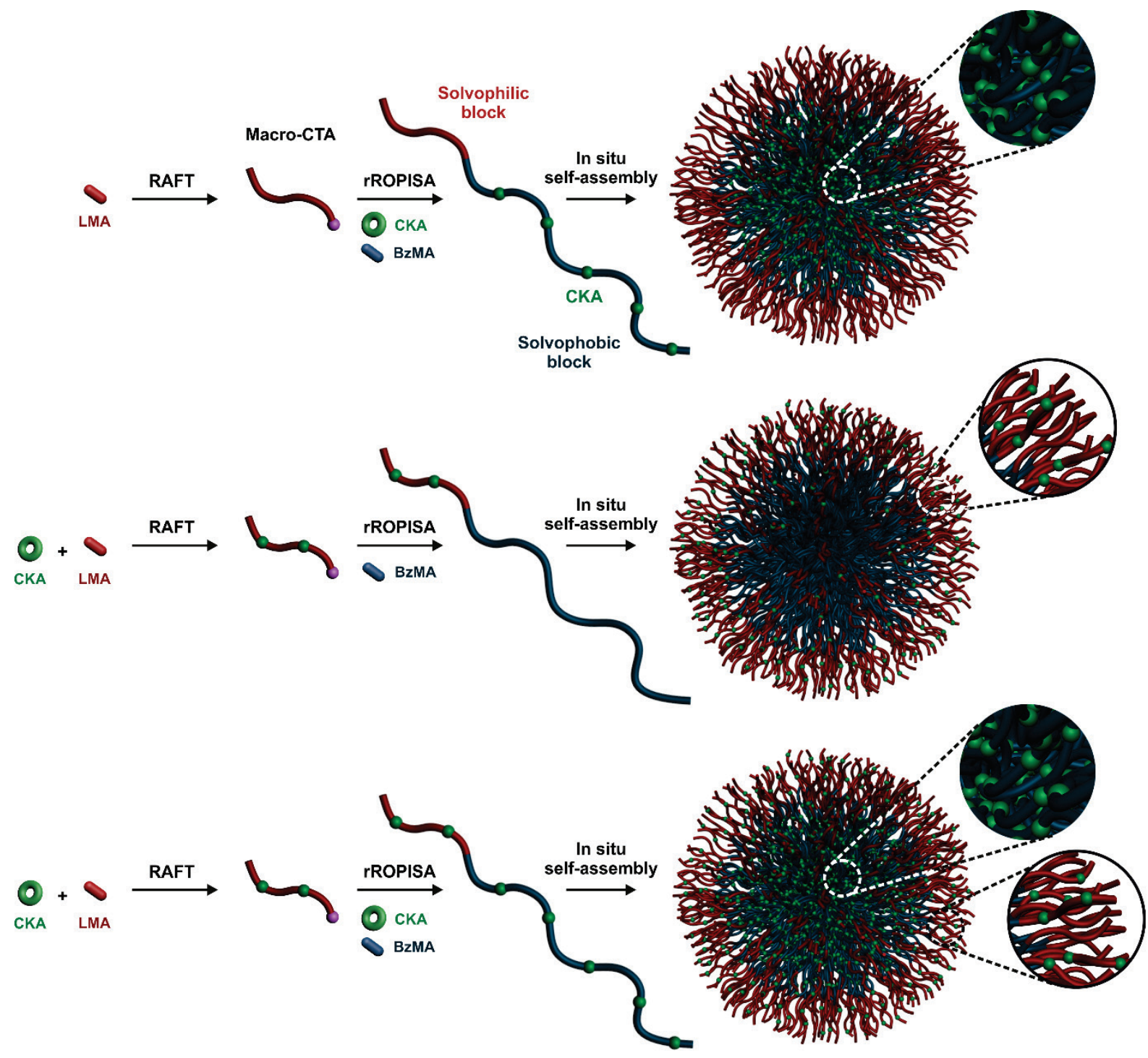

Figure 1. Synthesis of: (a) core-; (b) surface- and (c) surface plus core-degradable diblock copolymer nanoparticles by RAFT-mediated radical ring-opening copolymerization-induced self-assembly (rROPISA) from cyclic ketene acetals (CKA). LMA = lauryl methacrylate, BzMA = benzyl methacrylate. 


\section{Experimental part}

\section{Material}

Lauryl methacrylate (LMA, 96\%) and benzyl methacrylate (BzMA, 96\%) were purchased from Sigma-Aldrich and passed through basic alumina before use. Cumyl dithiobenzoate (CDB, 99\%), 4-cyano-4-[(dodecylsulfanylthiocarbonyl)sulfanyl]pentanoic acid (CDSPA, 97\%), anhydrous heptane, ruthenium(III) chloride hydrate $\left(\mathrm{RuCl}_{3}\right.$, Reagent Plus), sodium periodate (99.8\%) and 2,2'-azobis(2-methylpropionitrile) (AIBN, 98\%) were purchased from SigmaAldrich and used as received. Tert-butyl peroxy-2-ethylhexanoate (Trigonox 21S or T21s) initiator was purchased from AkzoNobel. Deuterated chloroform $\left(\mathrm{CDCl}_{3}\right)$ and tetrahydrofuran (TDF) were obtained from Eurisotop. 2-methylene-4-phenyl-1,3-dioxolane (MPDL), 2methylene-1,3-dioxepane (MDO) and 5,6-benzo-2-methylene-1,3-dioxepane (BMDO) were prepared as reported elsewhere using the cyclic bromoacetal as an intermediate. ${ }^{45}$ All other reactants were purchased from Sigma-Aldrich at the highest available purity and used as received and all other solvents were purchased from Carlo-Erba.

\section{Analytical method}

\subsection{Nuclear magnetic resonance (NMR) spectroscopy}

${ }^{1} \mathrm{H}$ NMR spectroscopy was performed in $5 \mathrm{~mm}$ diameter tubes in $\mathrm{CDCl}_{3}$ or $\mathrm{TDF}$ at $25{ }^{\circ} \mathrm{C}$ on a Bruker Avance 300 spectrometer at $300 \mathrm{MHz}$. The chemical shift scale was calibrated based on the internal solvent signal (chloroform: $\delta=7.26 \mathrm{ppm}$; tetrahydrofuran: $\delta=1.72$ and $3.58 \mathrm{ppm}$ ).

\subsection{Size exclusion chromatography (SEC)}

SEC was performed at $35^{\circ} \mathrm{C}$ with two columns from Polymer Laboratories (PL-gel MIXED$\mathrm{D} ; 300 \times 7.5 \mathrm{~mm}$; bead diameter, $5 \mu \mathrm{m}$; linear part, 400-400 $000 \mathrm{~g} . \mathrm{mol}^{-1}$ ) and a differential 
refractive index detector (Spectrasystem RI-150 from Thermo Electron Corp.), using chloroform $\left(\mathrm{CHCl}_{3}\right)$ as eluent at a flow rate of $1 \mathrm{~mL} \cdot \mathrm{min}^{-1}$ and toluene as a flow-rate marker. A conventional calibration curve was based on poly(methyl methacrylate) (PMMA) standards (peak molar masses, $M_{\mathrm{p}}=625-625500 \mathrm{~g} \cdot \mathrm{mol}^{-1}$ ) from Polymer Laboratories. This technique allowed $M_{\mathrm{n}}$ (number-average molar mass), $M_{\mathrm{w}}$ (weight-average molar mass), and $M_{\mathrm{w}} / M_{\mathrm{n}}$ (dispersity, $Ð$ ) to be determined. SEC of degraded copolymers was performed in the presence of $0.1 \%(\mathrm{v} / \mathrm{v})$ of trifluoroacetic acid (TFA, 99\%) in chloroform (in both the mobile phase and the sample) to avoid the formation of aggregates and/or interaction with the columns because of carboxylic acid chain ends.

\subsection{Dynamic light scattering (DLS)}

Nanoparticle diameter $\left(D_{z}\right)$ and particle size distribution (PSD) were measured by dynamic light scattering (DLS) with a Nano ZS from Malvern (173 ${ }^{\circ}$ scattering angle) at a temperature of 25 ${ }^{\circ} \mathrm{C}$. Copolymer dispersions were diluted in heptane $(80$ vol. $\%$, viscosity $=0.42 \mathrm{cP}, n=1.39$ at $\left.20^{\circ} \mathrm{C}\right)^{31}$ prior to light scattering studies.

\subsection{Transmission electron microscopy (TEM)}

The morphology of the nanoparticles was observed by TEM using a JEOL JEM-1400 operating at $80 \mathrm{kV}$. Images were acquired using an Orius camera (Gatan Inc, USA). $5 \mu \mathrm{L}$ of diluted block copolymer nanoparticle suspensions $(5 \%, \mathrm{v} / \mathrm{v})$ were deposited for $30 \mathrm{~s}$ on copper grids covered with formvar-carbon film. The excess solution was blotted off using a filter paper. Samples were then stained using ruthenium (IV) oxide for $7 \mathrm{~min}$ at $20^{\circ} \mathrm{C}$. This heavy metal compound acted as a positive stain to improve contrast. The ruthenium (IV) oxide was prepared as follows: ruthenium(III) chloride (30 mg) was added to water $(5 \mathrm{~mL})$ to form a black slurry; addition of 
sodium periodate $(200 \mathrm{mg})$ with stirring produced a yellow solution of ruthenium(IV) oxide within 5/10 min.

\subsection{Differential scanning calorimetry (DSC)}

DSC measurements were performed with a DSC Diamond Perkin-Elmer thermal analyzer equipped with Pyris thermal analysis software (version 9.1.0.0203, PerkinElmer), in the range of -20 to $100{ }^{\circ} \mathrm{C}$, at heating and cooling rates of $10{ }^{\circ} \mathrm{C} \mathrm{min}^{-1}$. Two consecutive scans were preceded by a 1 min isotherm at the initial temperature to allow the samples to set thermal equilibrium. In these conditions, repeated recordings were perfectly reproducible. All experiments were carried out in duplicate in hermetically sealed aluminum pans $(50 \mu \mathrm{L}$ total volume). Analyses were performed within the $2 \mathrm{~h}$ following sample conditioning (from 5.69 to $7.01 \mathrm{mg}$ total masses of copolymers). The reference was an empty aluminum pan. Apparatus calibration was performed with lauric acid $\left(99.95 \%\right.$ purity, $43.7^{\circ} \mathrm{C}$, melting temperature. The glass transition temperature $\left(T_{\mathrm{g}}\right)$ was determined by using the DSC apparatus software.

\section{Synthesis procedures}

\subsection{Synthesis of macro-RAFT agents}

\subsubsection{Synthesis of poly(lauryl methacrylate) using CDSPA as a RAFT agent (PLMA18-}

CDSPA). In a 40-mL vial, fitted with a rubber septum and a magnetic stirring bar, a mixture of $\operatorname{LMA}\left(2.10 \mathrm{~g}, 8.28 \times 10^{-3} \mathrm{~mol}\right), \operatorname{AIBN}\left(6.4 \mathrm{mg}, 3.90 \times 10^{-2} \mathrm{mmol}\right), \operatorname{CDSPA}\left(0.110 \mathrm{~g}, 2.74 \times 10^{-}\right.$ ${ }^{1} \mathrm{mmol}, \mathrm{LMA} / \mathrm{CDSPA} / \mathrm{AIBN}$ molar ratio $\left.=30 / 1 / 0.14\right)$ and anhydrous toluene $(2.5 \mathrm{~g}, 2.9 \mathrm{~mL})$ was degassed under stirring by argon bubbling for $15 \mathrm{~min}$ at room temperature. The mixture was then immersed in a preheated oil bath at $70{ }^{\circ} \mathrm{C}$ for $4 \mathrm{~h} .{ }^{1} \mathrm{H}$ NMR spectroscopy was used to determine LMA conversion (by integrating its two oxymethylene protons at 4.0-4.2 ppm) and 
$D P_{\mathrm{n}, \mathrm{NMR}}$ (by comparing the integrated signals of the two oxymethylene protons of PLMA at 4.0 ppm and the two protons in the $\alpha$-position to the carboxylic acid of CDSPA at $3.3 \mathrm{ppm}$ ). $D P_{\mathrm{n}, \mathrm{SEC}}, M_{\mathrm{n}}$ and $Ð$ were obtained by SEC. The copolymer was then precipitated twice in methanol and dried under high vacuum until constant weight.

\subsubsection{Synthesis of poly(lauryl methacrylate) using CDB as a RAFT agent (PLMA18-CDB).}

The previous procedure was repeated using CDB as a RAFT agent with the following modifications: the reaction mixture was composed of LMA $\left(1.01 \mathrm{~g}, 3.98 \times 10^{-3} \mathrm{~mol}\right)$, AIBN (5.5 mg, $\left.3.35 \times 10^{-2} \mathrm{mmol}\right), \mathrm{CDB}\left(0.047 \mathrm{~g}, 1.72 \times 10^{-1} \mathrm{mmol}, \mathrm{LMA} / \mathrm{CDB} / \mathrm{AIBN}\right.$ molar ratio $=$ 20/1/0.2) and anhydrous toluene (1.1 g, $1.3 \mathrm{~mL}) . D P_{\mathrm{n}, \mathrm{NMR}}$ was obtained by comparing the integrated signals of the two oxymethylene protons of PLMA at $4.0 \mathrm{ppm}$ and the ten protons from the aromatic rings of $\mathrm{CDB}$ at 7.1-8.1 $\mathrm{ppm}$.

\subsubsection{Synthesis of poly[(lauryl methacrylate)-co-(2-methylene-4-phenyl-1,3-dioxolane)]} using CDB as a RAFT agent (P(LMA-co-MPDL)-CDB). The synthesis of P(LMA17-coMPDL0.53)-CDB was as follows. In a 40-mL vial, fitted with a rubber septum and a magnetic stirring bar, a mixture of LMA (1.00 g, $\left.3.93 \times 10^{-3} \mathrm{~mol}\right)$, AIBN (6.8 mg, $\left.4.15 \times 10^{-2} \mathrm{mmol}\right)$, MPDL $\left(0.426 \mathrm{~g}, 2.63 \times 10^{-3} \mathrm{~mol}, f_{\mathrm{MPDL}, 0}=0.4\right), \mathrm{CDB}\left(0.049 \mathrm{~g}, 1.79 \times 10^{-1} \mathrm{mmol}\right.$, LMA/CDB/AIBN molar ratio $=20 / 1 / 0.2)$ and anhydrous toluene $(1.5 \mathrm{~g}, 1.7 \mathrm{~mL})$ was degassed under stirring by argon bubbling for $15 \mathrm{~min}$ at room temperature. The mixture was then immersed in a preheated oil bath at $70{ }^{\circ} \mathrm{C}$ for $4 \mathrm{~h} .{ }^{1} \mathrm{H}$ NMR spectroscopy was used to determine LMA conversion by integrating its two oxymethylene protons at 4.0-4.2 ppm. $D P_{\mathrm{n}, \mathrm{SEC}}, M_{\mathrm{n}}$ and $Ð$ were obtained by SEC. The copolymer was then precipitated twice in methanol and dried under high vacuum until constant weight. 
The same procedure was repeated with the following modifications to obtain P(LMA28co-MPDL 2.1)-CDB : the reaction mixture was composed of LMA (1.00 g, $\left.3.94 \times 10^{-3} \mathrm{~mol}\right)$, $\operatorname{AIBN}\left(3.4 \mathrm{mg}, 2.07 \times 10^{-2} \mathrm{mmol}\right), \operatorname{MPDL}\left(0.167 \mathrm{~g}, 1.03 \times 10^{-3} \mathrm{~mol}, f_{\mathrm{MPDL}, 0}=0.2\right), \mathrm{CDB}(0.027$ g, $9.89 \times 10^{-2} \mathrm{mmol}, \mathrm{LMA} / \mathrm{CDB} / \mathrm{AIBN}$ molar ratio $\left.=40 / 1 / 0.2\right)$ and anhydrous toluene $(1.2 \mathrm{~g}$, $1.4 \mathrm{~mL}$ ). The polymerization was performed at $90{ }^{\circ} \mathrm{C}$ for $4 \mathrm{~h}$.

\subsubsection{Synthesis of poly[(lauryl methacrylate)-co-(5,6-benzo-2-methylene-1,3-dioxepane)]} using CDB as a RAFT agent (P(LMA-co-BMDO)-CDB). The synthesis of P(LMA13-coBMDO0.7)-CDB was as follows. In a 40-mL vial, fitted with a rubber septum and a magnetic stirring bar, a mixture of LMA $\left(0.51 \mathrm{~g}, 2.00 \times 10^{-3} \mathrm{~mol}\right)$, AIBN $\left(2.8 \mathrm{mg}, 1.71 \times 10^{-2} \mathrm{mmol}\right)$, $\operatorname{BMDO}\left(0.207 \mathrm{~g}, 1.28 \times 10^{-3} \mathrm{~mol}, f_{\mathrm{BMDO}, 0}=0.4\right), \mathrm{CDB}\left(0.027 \mathrm{~g}, 1.00 \times 10^{-1} \mathrm{mmol}\right.$, $\mathrm{LMA} / \mathrm{CDB} / \mathrm{AIBN}$ molar ratio $=20 / 1 / 0.2)$ and anhydrous toluene $(0.7 \mathrm{~g}, 0.8 \mathrm{~mL})$ was degassed under stirring by argon bubbling for $15 \mathrm{~min}$ at room temperature. The mixture was then immersed in a preheated oil bath at $70{ }^{\circ} \mathrm{C}$ for $4 \mathrm{~h} .{ }^{1} \mathrm{H}$ NMR spectroscopy was used to determine LMA conversion by integrating its two oxymethylene protons at 4.0-4.2 ppm. $M_{\mathrm{n}}$ and $\oslash$ were obtained by SEC. The copolymer was then precipitated twice in methanol and dried under high vacuum until constant weight.

The synthesis of P(LMA21-co-BMDO1.1)-CDB was as follows. In a 40-mL vial, fitted with a rubber septum and a magnetic stirring bar, a mixture of LMA $\left(1.00 \mathrm{~g}, 3.94 \times 10^{-3} \mathrm{~mol}\right)$, AIBN $\left(6.2 \mathrm{mg}, 3.80 \times 10^{-2} \mathrm{mmol}\right), \operatorname{BMDO}\left(0.159 \mathrm{~g}, 9.81 \times 10^{-1} \mathrm{mmol}, f_{\mathrm{BMDO}, 0}=0.2\right), \mathrm{CDB}(0.057 \mathrm{~g}$, $2.10 \times 10^{-1} \mathrm{mmol}, \mathrm{LMA} / \mathrm{CDB} / \mathrm{AIBN}$ molar ratio $\left.=20 / 1 / 0.2\right)$ and anhydrous toluene $(1.2 \mathrm{~g}, 1.4$ $\mathrm{mL}$ ) was degassed under stirring by argon bubbling for $15 \mathrm{~min}$ at room temperature. The mixture was then immersed in a preheated oil bath at $70^{\circ} \mathrm{C}$ for $20 \mathrm{~h}$. 
The same procedure was repeated with: $f_{\mathrm{BMDO}, 0}=0.33$ to prepare $\left.\mathbf{P ( L M A 2 1 - c o - B M D O} \mathbf{1 . s}_{\mathbf{8}}\right)$ CDB [LMA (1.00 g, $\left.3.94 \times 10^{-3} \mathrm{~mol}\right), \operatorname{AIBN}\left(6.2 \mathrm{mg}, 3.78 \times 10^{-2} \mathrm{mmol}\right), \mathrm{BMDO}(0.331 \mathrm{~g}, 2.03$ $\left.\times 10^{-3} \mathrm{~mol}\right), \mathrm{CDB}\left(0.055 \mathrm{~g}, 2.03 \times 10^{-1} \mathrm{mmol}, \mathrm{LMA} / \mathrm{CDB} / \mathrm{AIBN}\right.$ molar ratio $\left.=20 / 1 / 0.2\right)$ and anhydrous toluene $(1.4 \mathrm{~g}, 1.6 \mathrm{~mL})] ; f_{\mathrm{BMDO}, 0}=0.4$ to prepare P(LMA14-co-BMDO1.4)-CDB [LMA (1.00 g, $\left.3.94 \times 10^{-3} \mathrm{~mol}\right), \operatorname{AIBN}\left(6.5 \mathrm{mg}, 3.98 \times 10^{-2} \mathrm{mmol}\right), \operatorname{BMDO}(0.430 \mathrm{~g}, 2.64 \times$ $\left.10^{-3} \mathrm{~mol}\right), \mathrm{CDB}\left(0.056 \mathrm{~g}, 2.05 \times 10^{-1} \mathrm{mmol}, \mathrm{LMA} / \mathrm{CDB} / \mathrm{AIBN}\right.$ molar ratio $\left.=20 / 1 / 0.2\right)$ and anhydrous toluene $(1.5 \mathrm{~g}, 1.7 \mathrm{~mL})] ; f_{\mathrm{BMDO}, 0}=0.5$ to prepare P(LMA16-co-BMDO1.7)-CDB [LMA (1.00 g, $\left.3.94 \times 10^{-3} \mathrm{~mol}\right), \operatorname{AIBN}\left(6.5 \mathrm{mg}, 3.98 \times 10^{-2} \mathrm{mmol}\right), \operatorname{BMDO}(0.659 \mathrm{~g}, 4.04 \times$ $\left.10^{-3} \mathrm{~mol}\right), \mathrm{CDB}\left(0.056 \mathrm{~g}, 2.05 \times 10^{-1} \mathrm{mmol}, \mathrm{LMA} / \mathrm{CDB} / \mathrm{AIBN}\right.$ molar ratio $\left.=20 / 1 / 0.2\right)$ and anhydrous toluene $(1.7 \mathrm{~g}, 2.0 \mathrm{~mL})] ; f_{\mathrm{BMDO}, 0}=0.6$ to prepare P(LMA17-co-BMDO2.7)-CDB [LMA (0.50 g, $\left.1.97 \times 10^{-3} \mathrm{~mol}\right), \operatorname{AIBN}\left(3.2 \mathrm{mg}, 1.97 \times 10^{-2} \mathrm{mmol}\right), \operatorname{BMDO}(0.481 \mathrm{~g}, 2.95 \times$ $\left.10^{-3} \mathrm{~mol}\right), \mathrm{CDB}\left(0.027 \mathrm{~g}, 9.92 \times 10^{-2} \mathrm{mmol}, \mathrm{LMA} / \mathrm{CDB} / \mathrm{AIBN}\right.$ molar ratio $\left.=20 / 1 / 0.2\right)$ and anhydrous toluene $(1.0 \mathrm{~g}, 1.1 \mathrm{~mL})]$ and $f_{\mathrm{BMDO}, 0}=0.7$ to prepare $\mathbf{P}(\mathbf{L M A 1 2}-$ co-BMDO3.9)-CDB [LMA (1.01 g, $\left.3.98 \times 10^{-3} \mathrm{~mol}\right), \operatorname{AIBN}\left(6.8 \mathrm{mg}, 4.15 \times 10^{-2} \mathrm{mmol}\right), \operatorname{BMDO}(1.470 \mathrm{~g}, 9.03 \times$ $\left.10^{-3} \mathrm{~mol}\right), \mathrm{CDB}\left(0.057 \mathrm{~g}, 2.11 \times 10^{-1} \mathrm{mmol}, \mathrm{LMA} / \mathrm{CDB} / \mathrm{AIBN}\right.$ molar ratio $\left.=20 / 1 / 0.2\right)$ and anhydrous toluene $(2.6 \mathrm{~g}, 3.1 \mathrm{~mL})]$.

The same procedure was also repeated with the following modifications to obtain P(LMA20-co-BMDO2.2)-CDB: the reaction mixture was composed of LMA (1.00 g, $3.93 \times 10^{-}$ $\left.{ }^{3} \mathrm{~mol}\right), \operatorname{AIBN}\left(3.2 \mathrm{mg}, 1.93 \times 10^{-2} \mathrm{mmol}\right), \mathrm{BMDO}\left(0.436 \mathrm{~g}, 2.67 \times 10^{-3} \mathrm{~mol}, f_{\mathrm{BMDO}, 0}=0.4\right)$, CDB $\left(0.0304 \mathrm{~g}, 1.12 \times 10^{-1} \mathrm{mmol}, \mathrm{LMA} / \mathrm{CDB} / \mathrm{AIBN}\right.$ molar ratio $\left.=40 / 1 / 0.2\right)$ and anhydrous toluene $(1.5 \mathrm{~g}, 1.7 \mathrm{~mL})$. The polymerization was performed at $90{ }^{\circ} \mathrm{C}$ for $4 \mathrm{~h}$.

\section{2 rROPISA procedures for core-degradable particles}


From now on, the following notations will be used for diblock copolymers: LMA $=\mathrm{L}$, BzMA $=\mathrm{Bz}, \mathrm{MDO}=\mathrm{M}, \mathrm{MPDL}=\mathrm{MP}, \mathrm{BMDO}=\mathrm{B}$. It gives $\mathbf{L}-\mathbf{B z}$ for PLMA- $b$-PBzMA, L-BzM for PLMA- $b$-P(BzMA-co-MDO), L-BzMP for PLMA- $b$-P(BzMA-co-MPDL), LMP-Bz for P(LMA-co-MPDL)- $b$-PBzMA, LB-Bz for P(LMA-co-BMDO)- $b$-PBzMA, LMP-BzMP for P(LMA-co-MPDL)- $b$-P(BzMA-co-MPDL) and LB-BzB for P(LMA-co-BMDO)- $b$-P(BzMAco-BMDO). When indicated, $\mathrm{x}$ in $\mathrm{Bz}_{\mathrm{x}}$ represents the targeted value at $100 \%$ monomer conversion.

\subsubsection{Synthesis of poly(lauryl methacrylate)-b-poly[(benzyl methacrylate)-co-(2-} methylene-1,3-dioxepane)] (PLMA-b-P(BzMA-co-MDO)) from PLMA18-CDSPA. A typical synthesis of PLMA $18-b-\mathrm{P}\left(\mathrm{BzMA}_{150}-c o-\mathrm{MDO}\right)\left(\mathbf{L}_{18}-\mathrm{Bz} 150 \mathrm{M}-\mathrm{CDSPA}\right)$ with $f_{\mathrm{MDO}, 0}=0.2$ by RAFT dispersion polymerization at $15 \mathrm{wt} . \%$ solids was as follows. In a $25-\mathrm{mL}$ round bottom flask, fitted with a rubber septum and a magnetic stirring bar, a mixture of BzMA $(0.677 \mathrm{~g}, 3.85$ $\left.\times 10^{-3} \mathrm{~mol}\right), \operatorname{MDO}\left(0.110 \mathrm{~g}, 9.65 \times 10^{-1} \mathrm{mmol}\right), \mathrm{T} 21 \mathrm{~s}$ initiator $\left(1.1 \mathrm{mg}, 5.09 \times 10^{-3} \mathrm{mmol}\right.$, dissolved at $0.1 \% \mathrm{w} / \mathrm{v}$ in heptane) and PLMA 18 -CDSPA (0.112 g, $2.24 \times 10^{-2} \mathrm{mmol}$; macro$\mathrm{CTA} /$ initiator molar ratio $=5.0)$ were dissolved in heptane $(5 \mathrm{~g}, 7.3 \mathrm{~mL})$. The mixture was degassed under stirring by argon bubbling for at least $15 \mathrm{~min}$ while immersed in an ice bath (to avoid solvent evaporation). The mixture was then immersed in a preheated oil bath at $90{ }^{\circ} \mathrm{C}$ for 20 h. Samples were periodically withdrawn to determine the BzMA conversion by ${ }^{1} \mathrm{H}$ NMR spectroscopy (by integrating the two oxymethylene protons of BzMA at 4.9 and $5.2 \mathrm{ppm}$ ) and the macromolecular characteristics, $M_{\mathrm{n}}$ and $\doteq$, by SEC. The nanoparticle colloidal characteristics, $D_{z}$ and PSD, were determined by DLS whereas their morphology was assessed by TEM. The nanoparticles were then dried under reduced pressure and solubilized in a minimal 
amount of dichloromethane (DCM) before precipitation in cold diethyl ether. The purified copolymer was collected after centrifugation $(10000 \mathrm{rpm}, 20 \mathrm{~min})$ and dried under high vacuum until constant weight.

The same procedure was also performed with $f_{\mathrm{MDO}, 0}=0.4$ [BzMA $\left(0.550 \mathrm{~g}, 3.13 \times 10^{-3}\right.$ $\mathrm{mol})$, MDO $\left(0.240 \mathrm{~g}, 2.10 \times 10^{-3} \mathrm{~mol}\right), \mathrm{T} 21 \mathrm{~s}$ initiator $\left(0.9 \mathrm{mg}, 4.17 \times 10^{-3} \mathrm{mmol}\right.$, dissolved at $0.1 \% \mathrm{w} / \mathrm{v}$ in heptane $)$ and PLMA 18 -CDSPA $\left(0.096 \mathrm{~g}, 1.92 \times 10^{-2} \mathrm{mmol}\right.$; macro-CTA/initiator molar ratio $=5.0)$ were dissolved in heptane $(5 \mathrm{~g}, 7.3 \mathrm{~mL})]$ and $f_{\mathrm{MDO}, 0}=0.7[\mathrm{BzMA}(0.339 \mathrm{~g}$, $\left.1.93 \times 10^{-3} \mathrm{~mol}\right), \operatorname{MDO}\left(0.500 \mathrm{~g}, 4.39 \times 10^{-3} \mathrm{~mol}\right), \mathrm{T} 21 \mathrm{~s}$ initiator $\left(0.5 \mathrm{mg}, 2.31 \times 10^{-3} \mathrm{mmol}\right.$, dissolved at $0.1 \% \mathrm{w} / \mathrm{v}$ in heptane) and $\mathrm{PLMA}_{18}$-CDSPA (0.058 g, $1.16 \times 10^{-2} \mathrm{mmol}$; macro$\mathrm{CTA} /$ initiator molar ratio $=5.0)$ were dissolved in heptane $(5 \mathrm{~g}, 7.3 \mathrm{~mL})]$.

\subsubsection{Synthesis of poly(lauryl methacrylate)-b-poly[(benzyl methacrylate)-co-(cyclic} ketene acetal] (PLMA-b-P(BzMA-co-CKA)) from PLMA18-CDB. A typical synthesis of PLMA $_{18}-b$-PBzMA $150\left(\mathbf{L}_{18-B z 150}\right)$ by RAFT dispersion polymerization at 15 wt.\% solids was as follows. A mixture of BzMA $\left(0.746 \mathrm{~g}, 4.24 \times 10^{-3} \mathrm{~mol}\right)$, T21s initiator $\left(1.2 \mathrm{mg}, 5.56 \times 10^{-3}\right.$ mmol, dissolved at $0.1 \% \mathrm{w} / \mathrm{v}$ in heptane $)$ and $\operatorname{PLMA}_{18}$-CDB $\left(0.136 \mathrm{~g}, 2.78 \times 10^{-2} \mathrm{mmol}\right.$; macroCTA/initiator molar ratio $=5.0)$ were dissolved in heptane $(5 \mathrm{~g}, 7.3 \mathrm{~mL})$. The solution was transferred into a $25-\mathrm{mL}$ round bottom flask, fitted with a rubber septum and a magnetic stirring bar. The flask was then degassed under stirring by argon bubbling for at least $15 \mathrm{~min}$ and immersed in a preheated oil bath at $90^{\circ} \mathrm{C}$ for $20 \mathrm{~h}$. The workup procedure was identical to that described in section 3.2.1.

The same procedure was also performed with $f_{\mathrm{MDO}, 0}=0.2$ to prepare $\mathrm{PLMA}_{18}-b$ $\mathrm{P}\left(\mathrm{BzMA}_{150}-\mathrm{co}-\mathrm{MDO}\right)(\mathbf{L} 18-\mathrm{Bz} 150 \mathbf{M})$ [BzMA (0.665 g, $\left.3.78 \times 10^{-3} \mathrm{~mol}\right), \mathrm{MDO}(0.113 \mathrm{~g}, 9.91 \times$ 
$\left.10^{-4} \mathrm{~mol}\right), \mathrm{T} 21 \mathrm{~s}$ initiator $\left(1.1 \mathrm{mg}, 5.09 \times 10^{-3} \mathrm{mmol}\right.$, dissolved at $0.1 \% \mathrm{w} / \mathrm{v}$ in heptane $)$ and $\mathrm{PLMA}_{18}$-CDB $\left(0.119 \mathrm{~g}, 2.44 \times 10^{-2} \mathrm{mmol}\right.$; macro-CTA/initiator molar ratio $\left.=5.0\right)$ were dissolved in heptane $(5 \mathrm{~g}, 7.3 \mathrm{~mL})]$ and with $f_{\mathrm{MPDL}, 0}=0.2$ to prepare $\mathrm{PLMA}_{18}-b-\mathrm{P}\left(\mathrm{BzMA}_{150^{-}}\right.$ co-MPDL) (L18-Bz150MP) [BzMA (1.230 g, $\left.6.99 \times 10^{-3} \mathrm{~mol}\right)$, MPDL $\left(0.282 \mathrm{~g}, 1.74 \times 10^{-3}\right.$ mol), T21s initiator (2.0 mg, $9.27 \times 10^{-3} \mathrm{mmol}$, dissolved at $0.1 \% \mathrm{w} / \mathrm{v}$ in heptane $)$ and PLMA $18^{-}$ $\mathrm{CDB}\left(0.261 \mathrm{~g}, 5.32 \times 10^{-2} \mathrm{mmol}\right.$; macro-CTA/initiator molar ratio $\left.=5.0\right)$ were dissolved in heptane $(10 \mathrm{~g}, 14.6 \mathrm{~mL})]$.

\section{3 rROPISA procedures for surface-degradable particles}

\subsubsection{Synthesis of poly[(lauryl methacrylate)-co-(2-methylene-4-phenyl-1,3-dioxolane)]-b-}

poly(benzyl methacrylate) (P(LMA-co-MPDL)-b-PBzMA). A typical synthesis of P(LMA $\left.{ }_{17}-c o-\mathrm{MPDL}_{0.53}\right)-b$-PBzMA $150\left(\mathbf{L}_{17} \mathbf{M P}_{\mathbf{0 . 5 3}}-\mathrm{Bz}_{150}\right)$ by RAFT dispersion polymerization at $15 \mathrm{wt} . \%$ solids was as follows. In a $25-\mathrm{mL}$ round bottom flask, fitted with a rubber septum and a magnetic stirring bar, a mixture of BzMA $\left(0.383 \mathrm{~g}, 2.18 \times 10^{-3} \mathrm{~mol}\right)$, T21s initiator $(0.6$ $\mathrm{mg}, 2.78 \times 10^{-3} \mathrm{mmol}$, dissolved at $0.1 \% \mathrm{w} / \mathrm{v}$ in heptane $)$ and $\mathrm{P}\left(\mathrm{LMA}_{17}-\mathrm{co}-\mathrm{MPDL}_{0.53}\right)-\mathrm{CDB}$ $\left(0.068 \mathrm{~g}, 1.45 \times 10^{-2} \mathrm{mmol}\right.$; macro-CTA/initiator molar ratio $\left.=5.0\right)$ were dissolved in heptane $(2.5 \mathrm{~g}, 3.7 \mathrm{~mL})$. The mixture was degassed under stirring by argon bubbling for at least $15 \mathrm{~min}$ while immersed in an ice bath (to avoid solvent evaporation). The mixture was then immersed in a preheated oil bath at $90{ }^{\circ} \mathrm{C}$ for $20 \mathrm{~h}$. The workup procedure was identical to that described in section 3.2.1.

The same procedure was repeated with the following modifications for the synthesis of P(LMA $\left.28-c o-\mathrm{MPDL}_{2.1}\right)-b$-PBzMA 150 (L28MP2.1-Bz150): the mixture was composed of BzMA $\left(0.343 \mathrm{~g}, 1.95 \times 10^{-3} \mathrm{~mol}\right)$, T21s initiator $\left(0.6 \mathrm{mg}, 2.78 \times 10^{-3} \mathrm{mmol}\right.$, dissolved at $0.1 \% \mathrm{w} / \mathrm{v}$ in 
heptane $)$ and $\mathrm{P}\left(\mathrm{LMA}_{28}-\mathrm{co}-\mathrm{MPDL}_{2.1}\right)$-CDB $\left(0.098 \mathrm{~g}, 1.29 \times 10^{-2} \mathrm{mmol}\right.$; macro-CTA/initiator molar ratio $=5.0)$ were dissolved in heptane $(2.5 \mathrm{~g}, 3.7 \mathrm{~mL})$. The mixture was then immersed in a preheated oil bath at $90{ }^{\circ} \mathrm{C}$ for $16 \mathrm{~h}$.

\subsubsection{Synthesis of poly[(lauryl methacrylate)-co-(5,6-benzo-2-methylene-1,3-dioxepane)]-} b-poly(benzyl methacrylate) (P(LMA-co-BMDO)-b-PBzMA)). A typical synthesis of P(LMA $\left.21-c o-\mathrm{BMDO}_{1.1}\right)-b$-PBzMA 75 (L21 $\left.\mathbf{B}_{1.1}-\mathbf{B z}_{75}\right)$ by RAFT dispersion polymerization at 15 wt.\% solids was as follows. In a $25-\mathrm{mL}$ round bottom flask, fitted with a rubber septum and a magnetic stirring bar, a mixture of BzMA $\left(0.624 \mathrm{~g}, 3.55 \times 10^{-3} \mathrm{~mol}\right)$, T21s initiator $(2 \mathrm{mg}, 9.26$ $\times 10^{-3} \mathrm{mmol}$, dissolved at $0.1 \% \mathrm{w} / \mathrm{v}$ in heptane $)$ and $\mathrm{P}\left(\mathrm{LMA}_{21}-\mathrm{co}-\mathrm{BMDO}_{1.1}\right)-\mathrm{CDB}(0.266 \mathrm{~g}$, $4.59 \times 10^{-2} \mathrm{mmol}$; macro-CTA/initiator molar ratio $\left.=5.0\right)$ were dissolved in heptane $(5 \mathrm{~g}, 7.3$ $\mathrm{mL}$ ). The mixture was degassed under stirring by argon bubbling for at least $15 \mathrm{~min}$ while immersed in an ice bath (to avoid solvent evaporation). The mixture was then immersed in a preheated oil bath at $90{ }^{\circ} \mathrm{C}$ for $8 \mathrm{~h}$. The workup procedure was identical to that described in section 3.2.1.

The same procedure was also repeated with $\mathrm{PLMA}_{18}-\mathrm{CDB}$ to prepare $\mathrm{PLMA}_{18}-b-\mathrm{PBzMA}_{75}$ (L18-Bz75) [BzMA (0.657 g, $\left.3.7 \times 10^{-3} \mathrm{~mol}\right)$, T21s initiator $\left(2 \mathrm{mg}, 9.72 \times 10^{-3} \mathrm{mmol}\right.$, dissolved at $0.1 \% \mathrm{w} / \mathrm{v}$ in heptane $)$ and $\mathrm{PLMA}_{18} \mathrm{CDB}\left(0.233 \mathrm{~g}, 4.76 \times 10^{-2} \mathrm{mmol}\right.$; macro-CTA/initiator molar ratio $=5.0)$ dissolved in heptane $(5 \mathrm{~g}, 7.3 \mathrm{~mL})]$; with $\mathrm{P}\left(\mathrm{LMA}_{21}-\mathrm{co}-\mathrm{BMDO}_{1.8}\right)-\mathrm{CDB}$ to

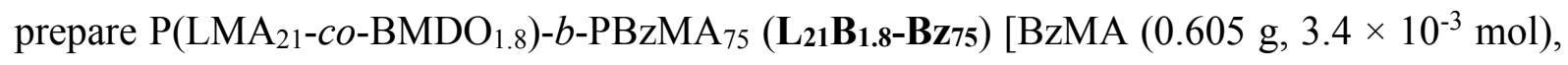
T21s initiator $\left(2 \mathrm{mg}, 9.26 \times 10^{-3} \mathrm{mmol}\right.$, dissolved at $0.1 \% \mathrm{w} / \mathrm{v}$ in heptane $)$ and $\mathrm{P}\left(\mathrm{LMA}_{21}-\mathrm{co}-\right.$ $\left.\mathrm{BMDO}_{1.8}\right)-\mathrm{CDB}\left(0.276 \mathrm{~g}, 4.68 \times 10^{-2} \mathrm{mmol}\right.$; macro-CTA/initiator molar ratio $\left.=5.0\right)$ dissolved in heptane $(5 \mathrm{~g}, 7.3 \mathrm{~mL})]$; with $\mathrm{P}\left(\mathrm{LMA}_{14}-c o-\mathrm{BMDO}_{1.4}\right)-\mathrm{CDB}$ to prepare $\mathrm{P}\left(\mathrm{LMA}_{14}-\mathrm{co}\right.$ - 
$\left.\mathrm{BMDO}_{1.4}\right)-b-\mathrm{PBzMA}_{75}\left(\mathbf{L}_{\mathbf{1 4}} \mathbf{B}_{\mathbf{1 . 4}}-\mathbf{B z}_{75}\right)$ [BzMA (0.672 g, $\left.3.8 \times 10^{-3} \mathrm{~mol}\right)$, T21s initiator $(2.2 \mathrm{mg}$, $1.03 \times 10^{-2} \mathrm{mmol}$, dissolved at $0.1 \% \mathrm{w} / \mathrm{v}$ in heptane $)$ and $\mathrm{P}\left(\mathrm{LMA}_{14}-\mathrm{co}-\mathrm{BMDO}_{1.4} \mathrm{CDB}\right)(0.205$ g, $5.00 \times 10^{-2} \mathrm{mmol}$; macro-CTA/initiator molar ratio $\left.=5.0\right)$ dissolved in heptane $\left.(5 \mathrm{~g}, 7.3 \mathrm{~mL})\right]$; with $\mathrm{P}\left(\mathrm{LMA}_{16}-c o-\mathrm{BMDO}_{1.7}\right)-\mathrm{CDB}$ to prepare $\mathrm{P}\left(\mathrm{LMA}_{16}-c o-\mathrm{BMDO}_{1.7}\right)-b-\mathrm{PBzMA}_{75}$ (L16 $\mathbf{B}_{1.7-}$ Bz75) [BzMA (0.664 g, $\left.3.8 \times 10^{-3} \mathrm{~mol}\right)$, T21s initiator $\left(2.1 \mathrm{mg}, 9.72 \times 10^{-3} \mathrm{mmol}\right.$, dissolved at $0.1 \% \mathrm{w} / \mathrm{v}$ in heptane $)$ and $\mathrm{P}\left(\mathrm{LMA}_{16}-\mathrm{co}-\mathrm{BMDO}_{1.7} \mathrm{CDB}\right)\left(0.223 \mathrm{~g}, 4.96 \times 10^{-2} \mathrm{mmol}\right.$; macro$\mathrm{CTA} /$ initiator molar ratio $=5.0)$ dissolved in heptane $(5 \mathrm{~g}, 7.3 \mathrm{~mL})]$ and with $\mathrm{P}\left(\mathrm{LMA}_{12}-\mathrm{co}\right.$ $\left.\mathrm{BMDO}_{3.9}\right)$-CDB to prepare $\mathrm{P}\left(\mathrm{LMA}_{12}-c o-\mathrm{BMDO}_{3.9}\right)-b-\mathrm{PBzMA}_{75}\left(\mathbf{L}_{\mathbf{1 2}} \mathbf{B}_{3.9}-\mathbf{B z} 75\right)$ [BzMA (0.682 $\left.\mathrm{g}, 3.9 \times 10^{-3} \mathrm{~mol}\right)$, T21s initiator $\left(2.2 \mathrm{mg}, 1.02 \times 10^{-2} \mathrm{mmol}\right.$, dissolved at $0.1 \% \mathrm{w} / \mathrm{v}$ in heptane $)$ and $\mathrm{P}\left(\mathrm{LMA}_{12}-\mathrm{co}-\mathrm{BMDO}_{3.9}\right)-\mathrm{CDB}\left(0.214 \mathrm{~g}, 5.10 \times 10^{-2} \mathrm{mmol}\right.$; macro-CTA/initiator molar ratio $=5.0)$ dissolved in heptane $(5 \mathrm{~g}, 7.3 \mathrm{~mL})]$.

The same procedure was also repeated with the following modifications for the synthesis of $\mathrm{P}\left(\mathrm{LMA}_{20}-c o-\mathrm{BMDO}_{2.2}\right)-b-\mathrm{PBzMA}_{75}\left(\mathbf{L}_{20} \mathbf{B}_{2.2}-\mathbf{B z}_{75}\right)$ : the mixture was composed of BzMA $\left(0.311 \mathrm{~g}, 1.77 \times 10^{-3} \mathrm{~mol}\right)$, T21s initiator $\left(1.0 \mathrm{mg}, 4.63 \times 10^{-3} \mathrm{mmol}\right.$, dissolved at $0.1 \% \mathrm{w} / \mathrm{v}$ in heptane $)$ and $\mathrm{P}\left(\mathrm{LMA}_{20}-\mathrm{co}-\mathrm{BMDO}_{2.2}\right)-\mathrm{CDB}\left(0.134 \mathrm{~g}, 2.36 \times 10^{-2} \mathrm{mmol}\right.$; macro-CTA/initiator molar ratio $=5.0)$ were dissolved in heptane $(2.5 \mathrm{~g}, 3.7 \mathrm{~mL})$. The mixture was then immersed in a preheated oil bath at $90{ }^{\circ} \mathrm{C}$ for $16 \mathrm{~h}$.

\section{4 rROPISA procedures for surface- and co-degradable particles}

3.4.1 Synthesis of poly[(lauryl methacrylate)-co-(2-methylene-4-phenyl-1,3-dioxolane)]-bpoly[(benzyl methacrylate)-co-(2-methylene-4-phenyl-1,3-dioxolane)] (P(LMA-coMPDL)-b-P(BzMA-co-MPDL)). A typical synthesis of $\quad$ P(LMA28-co-MPDL 2.1$)-b-$ P(BzMA $150-c o-M P D L)$ (L28MP2.1-Bz150MP) by RAFT dispersion polymerization at 15 wt.\% 
solids was as follows. In a $25-\mathrm{mL}$ round bottom flask, fitted with a rubber septum and a magnetic stirring bar, a mixture of BzMA (0.241 g, $\left.1.37 \times 10^{-3} \mathrm{~mol}\right)$, MPDL $\left(0.142 \mathrm{~g}, 8.8 \times 10^{-}\right.$ $\left.{ }^{1} \mathrm{mmol}, f_{\mathrm{MPDL}, 0}=0.4\right), \mathrm{T} 21 \mathrm{~s}$ initiator $\left(0.4 \mathrm{mg}, 1.85 \times 10^{-3} \mathrm{mmol}\right.$, dissolved at $0.1 \% \mathrm{w} / \mathrm{v}$ in heptane $)$ and $\mathrm{P}\left(\mathrm{LMA}_{28}-\mathrm{co}-\mathrm{MPDL}_{2.1}\right)$-CDB $\left(0.067 \mathrm{~g}, 8.82 \times 10^{-3} \mathrm{mmol}\right.$; macro-CTA/initiator molar ratio $=5.0)$ were dissolved in heptane $(2.5 \mathrm{~g}, 3.7 \mathrm{~mL})$. The mixture was degassed under stirring by argon bubbling for at least 15 min while immersed in an ice bath (to avoid solvent evaporation). The mixture was then immersed in a preheated oil bath at $90{ }^{\circ} \mathrm{C}$ for $16 \mathrm{~h}$. The workup procedure was identical to that described in section 3.2.1.

\subsubsection{Synthesis of poly[(lauryl methacrylate)-co-(5,6-benzo-2-methylene-1,3-dioxepane)]-} b-poly[(benzyl methacrylate)-co-(5,6-benzo-2-methylene-1,3-dioxepane)] (P(LMA-coBMDO)- $\boldsymbol{b}$-P(BzMA-co-BMDO)). A typical synthesis of $\mathrm{P}\left(\mathrm{LMA}_{20}-c o-\mathrm{BMDO}_{2.2}\right)-b-$ P(BzMA75-co-BMDO) ( $\left.\mathbf{L}_{20} \mathbf{B}_{2.2}-\mathbf{B z}_{75} \mathbf{B}\right)$ by RAFT dispersion polymerization at 15 wt.\% solids was as follows. In a $25-\mathrm{mL}$ round bottom flask, fitted with a rubber septum and a magnetic stirring bar, a mixture of BzMA $\left(0.217 \mathrm{~g}, 1.23 \times 10^{-3} \mathrm{~mol}\right)$, BMDO $\left(0.150 \mathrm{~g}, 9.3 \times 10^{-1} \mathrm{mmol}\right.$, $\left.f_{\text {MPDL }, 0}=0.4\right)$, T21s initiator $\left(0.7 \mathrm{mg}, 3.24 \times 10^{-3} \mathrm{mmol}\right.$, dissolved at $0.1 \% \mathrm{w} / \mathrm{v}$ in heptane $)$ and $\mathrm{P}\left(\mathrm{LMA}_{20}-\mathrm{co}-\mathrm{BMDO}_{2.2}\right)-\mathrm{CDB}\left(0.094 \mathrm{~g}, 1.65 \times 10^{-2} \mathrm{mmol}\right.$; macro-CTA/initiator molar ratio = 5.0) were dissolved in heptane $(2.5 \mathrm{~g}, 3.7 \mathrm{~mL})$. The mixture was degassed under stirring by argon bubbling for at least $15 \mathrm{~min}$ while immersed in an ice bath (to avoid solvent evaporation). The mixture was then immersed in a preheated oil bath at $90{ }^{\circ} \mathrm{C}$ for $16 \mathrm{~h}$. The workup procedure was identical to that described in section 3.2.1. 


\section{Degradation of the copolymers}

In a 5-mL vial, $30 \mathrm{mg}$ of purified copolymer was dissolved in $1.5 \mathrm{~mL}$ of THF. After solubilization, $1.5 \mathrm{~mL}$ of potassium hydroxide solution $(\mathrm{KOH}, 5 \mathrm{wt} . \%)$ in methanol was added. The cloudy mixture was stirred at room temperature. Samples $(1 \mathrm{~mL})$ were periodically taken, immediately dried under vacuum and mixed with $2 \mathrm{~mL}$ of chloroform, allowing salts filtration by using $0.2 \mu \mathrm{m}$ PTFE filters. $\mathrm{HCl}$ solution $\left(1 \mathrm{~mL}, 1 \mathrm{~mol} . \mathrm{L}^{-1}\right)$ was then added to the organic solution followed by extraction of the aqueous layer (repeated three times). Solvent was then removed under reduced pressure and degradation products were analyzed by SEC.

The theoretical $M_{\mathrm{n}}$ after degradation, $M_{\mathrm{n}, \mathrm{th}}{ }^{\infty}$, is calculated according to Eq. 1 .

$M_{\mathrm{n}, \mathrm{th}}{ }^{\infty}=\mathrm{MW}_{\mathrm{BzMA}} \times\left(1 / F_{\mathrm{CKA}}-1\right)+\mathrm{MW}_{\mathrm{CKA}}$

Eq. 1

$M_{\mathrm{n}}$ decrease in $\%$ after hydrolytic degradation of the purified copolymers is calculated according to Eq. 2.

$M_{\mathrm{n}}$ decrease $(\%)=\left(\exp \cdot M_{\mathrm{n}, \mathrm{SEC}}-\right.$ initial $\left.M_{\mathrm{n}, \mathrm{SEC}}\right) /$ initial $M_{\mathrm{n}, \mathrm{SEC}}$

Eq. 2

\section{Results and Discussion}

\section{Application of rROPISA to MDO}

Similarly to our previous rROPISA experiments with MPDL and BMDO as CKAs, ${ }^{31}$ we first synthesized a well-defined PLMA 18 -macro RAFT agent $\left(M_{\mathrm{n}, \mathrm{SEC}}=5000\right.$ g.mol $\left.{ }^{-1}, Ð=1.13\right)$ by polymerization of LMA at $70^{\circ} \mathrm{C}$ for $4 \mathrm{~h}$ in anhydrous toluene using CDSPA as a RAFT agent and AIBN as an initiator (see Table S1 and Figure S1 and S2). It was then chain extended by 
copolymerizing BzMA (targeted $\left.D P_{\mathrm{n}, \mathrm{BzMA}}=150\right)$ with a variable amount of $\mathrm{MDO}\left(f_{\mathrm{MDO}, 0}=0\right.$ 0.7 ) at $15 \mathrm{wt} . \%$ solids in heptane for $20 \mathrm{~h}$ at $90^{\circ} \mathrm{C}$ using T21s as an initiator (Figure 2).
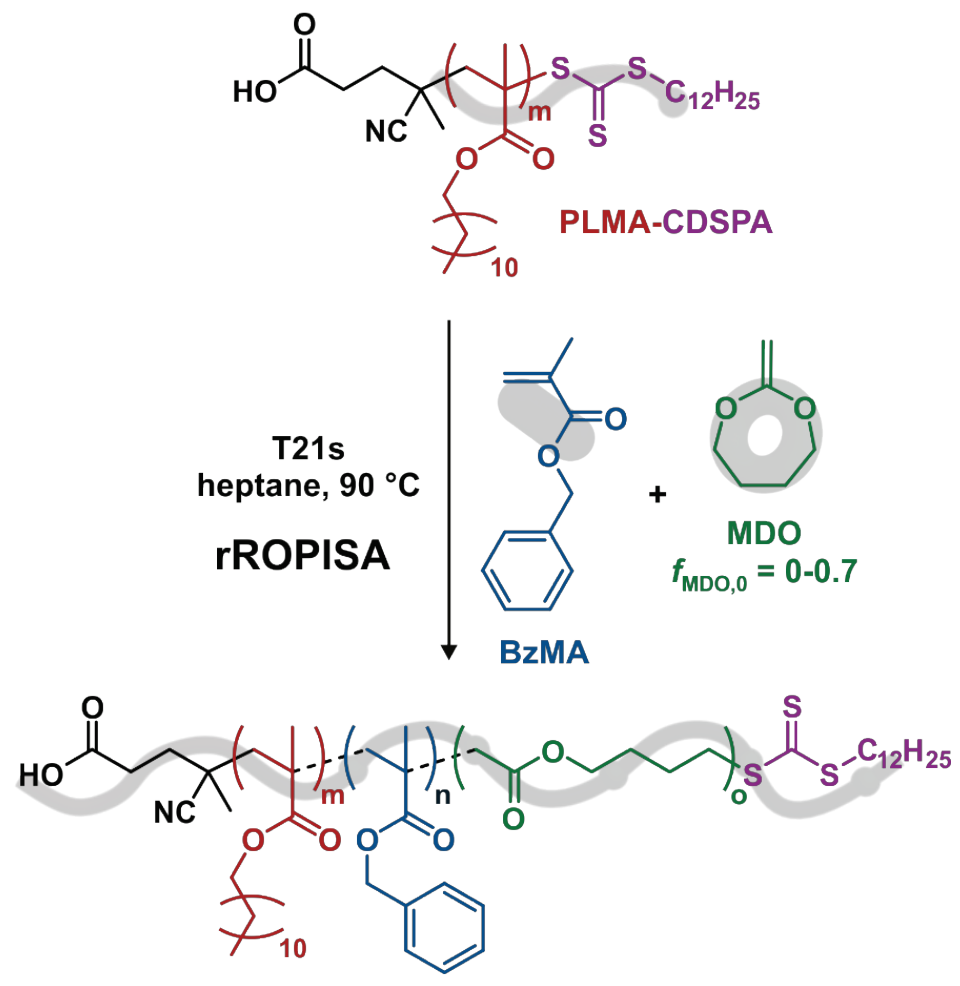

\section{PLMA-b-P(BzMA-co-MDO)}

Figure 2. RAFT-mediated synthesis of poly(lauryl methacrylate) (PLMA) followed by radical ring-opening copolymerization-induced self-assembly (rROPISA) of benzyl methacrylate (BzMA) and 2-methylene-1,3-dioxepane (MDO) to synthesize PLMA- $b$-P(BzMA-co-MDO) (L-BzM) diblock copolymer nanoparticles.

Adding MDO to the reaction medium decreased the polymerization rate compared to BzMA homopolymerization as the higher $f_{\mathrm{MDO}, 0}$, the lower the BzMA conversion (conv. $=90 \%$ and $52 \%$ for $f_{\mathrm{MDO}, 0}=0$ and 0.7 , respectively) (Figure $3 \mathrm{a}$ and Table S2). Although this trend was already observed with MPDL and BMDO under identical experimental conditions, ${ }^{31}$ the effect of MDO on the polymerization rate was more pronounced. Nevertheless, all $M_{\mathrm{n}}$ values linearly increased with the monomer conversion in good agreement with the theoretical $M_{\mathrm{n}}$ up to $\sim 50 \%$ 
monomer conversion where $D$ reached $\sim 2$ (Figure $3 b$ ). Increasing further the monomer conversion led however to some deviation in the values of experimental and theoretical $M_{\mathrm{n}}$, together with a significant increase in the dispersity for $f_{\mathrm{MDO}, 0}=0.4$ and 0.7 , which testified for a loss of control for high initial amounts of MDO. This observation is likely assigned to occurrence of termination reactions resulting from fragmentation of the radical intermediate via the Z-group, as already observed with MDO. ${ }^{46}$ Note that the control could be likely improved by investigating other RAFT agents. ${ }^{47}$ In comparison, the synthesis of MPDL- and BMDOcontaining copolymers obtained under the same rROPISA conditions were better controlled $(\bigoplus$ $=1.14-1.62)$.

(a)

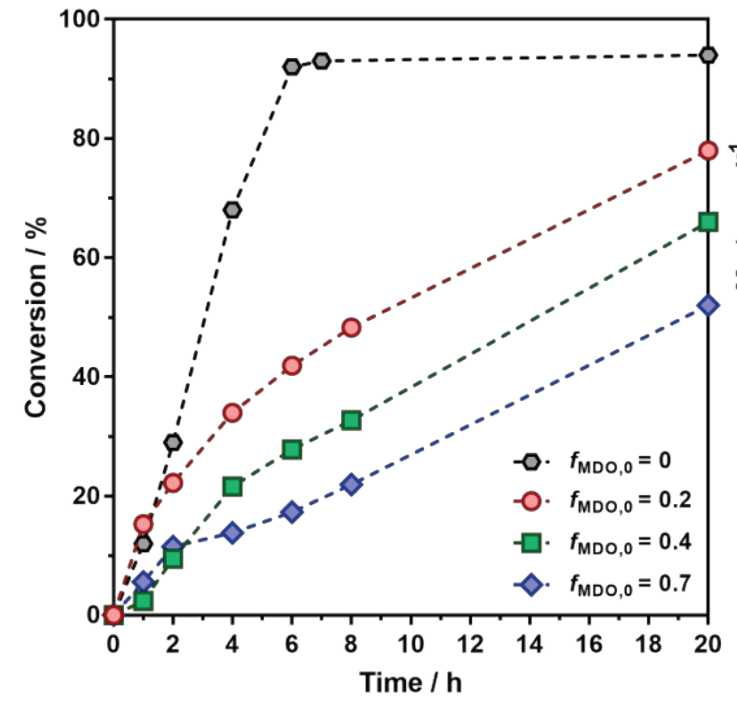

(b)

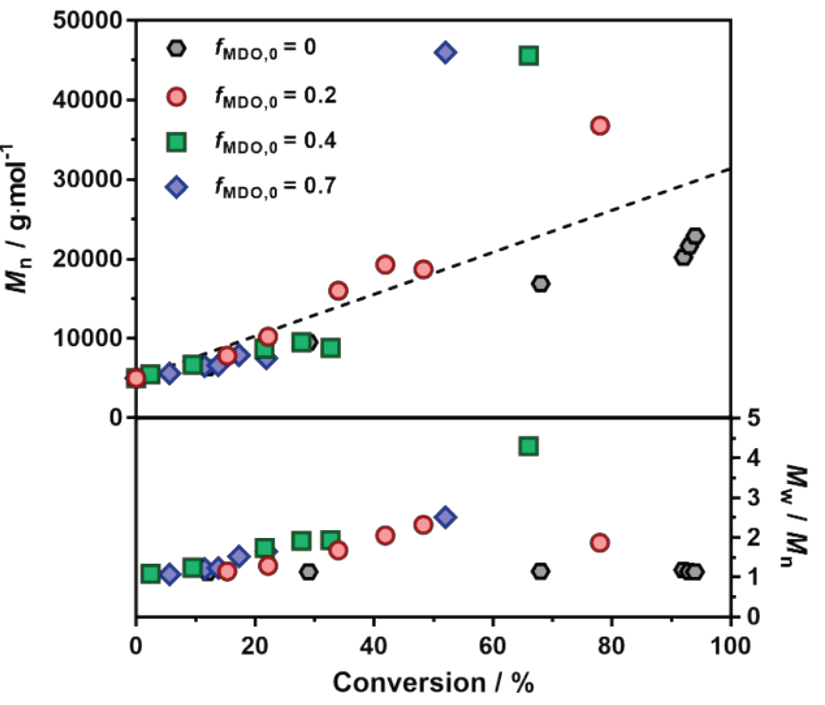

Figure 3. RAFT-mediated dispersion copolymerization of benzyl methacrylate (BzMA) and 2methylene-1,3-dioxepane (MDO) initiated by a poly(lauryl methacrylate) ${ }_{18}$ macro-RAFT agent at $15 \mathrm{wt} . \%$ solids in heptane at $90{ }^{\circ} \mathrm{C}$ as a function of the initial fraction of $\mathrm{MDO}\left(f_{\mathrm{MDO}, 0}=0\right.$ 0.7). (a) BzMA conversion vs. time. Dashed lines connecting data points are guides for the eye only. (b) Number-average molar mass $\left(M_{\mathrm{n}}\right)$ and dispersity $\left(M_{\mathrm{w}} / M_{\mathrm{n}}\right)$ vs. BzMA conversion determined by SEC. The dashed black line represents the theoretical $M_{\mathrm{n}}$. 
Nonetheless, ${ }^{1} \mathrm{H}$ NMR spectroscopy of the purified copolymers obtained after precipitation in cold diethyl ether not only revealed the presence of MDO into the copolymer structure (see protons $g, h$ and $i$ in Figure $4 a)$, but also its increasing amount in the copolymer $\left(F_{\mathrm{MDO}}\right)$ when $f_{\mathrm{MDO}, 0}$ was increased. Indeed, $F_{\mathrm{MDO}}=0.04,0.10$ and 0.19 for $f_{\mathrm{MDO}, 0}=0.2,0.4$ and 0.7 , respectively. Interestingly, $F_{\mathrm{MDO}}$ values were comparable to those obtained with MPDL and BMDO under identical rROPISA conditions. ${ }^{31}$ Hydrolytic degradation of the different copolymers under accelerated conditions (i.e., THF/MeOH, 2.5 wt.\% KOH) was then performed to assess the presence of ester group into the solvophobic block (Figure 4b). MDOfree $\mathbf{L}_{18}-\mathbf{B z} 150$ copolymer $\left(F_{\mathrm{MDO}}=0\right)$ showed no sign of degradation as attested by its constant $M_{\mathrm{n}}$ over time. ${ }^{31}$ Conversely, L18-Bz150M copolymers led to rapid and significant degradation in good correlation with their respective amounts of MDO (the higher $F_{\mathrm{MDO}}$, the greater the degradation), as shown by the shifts of their SEC chromatograms towards lower $M_{\mathrm{n}}$ values (Figure S3), and thus the decrease of the $M_{\mathrm{n}}$ over time (Figure 4b). According to SEC analysis, the $M_{\mathrm{n}}$ after degradation reached $5800 \mathrm{~g} \cdot \mathrm{mol}^{-1}\left(-82 \% M_{\mathrm{n}}\right.$ loss $)$ for $F_{\mathrm{MDO}}=0.04,3000 \mathrm{~g} \cdot \mathrm{mol}^{-1}$ $\left(-92 \% M_{\mathrm{n}}\right.$ loss $)$ for $F_{\mathrm{MDO}}=0.10$, and $\sim 100 \mathrm{~g} \cdot \mathrm{mol}^{-1}\left(-99 \% M_{\mathrm{n}}\right.$ loss $)$ for $F_{\mathrm{MDO}}=0.19$, respectively. $M_{\mathrm{n}}$ of, these values are rather close to the theoretical $M_{\mathrm{n}}$ after degradation as $M_{\mathrm{n}, \mathrm{th}}$ ${ }^{\infty}=4300,1700$ and 870 g.mol ${ }^{-1}$, respectively. However, given the DRI detector response may vary for low molar mass compounds, these results and calculations, especially for the fully degraded copolymers, must be taken with precaution and can only be considered as indicative. 


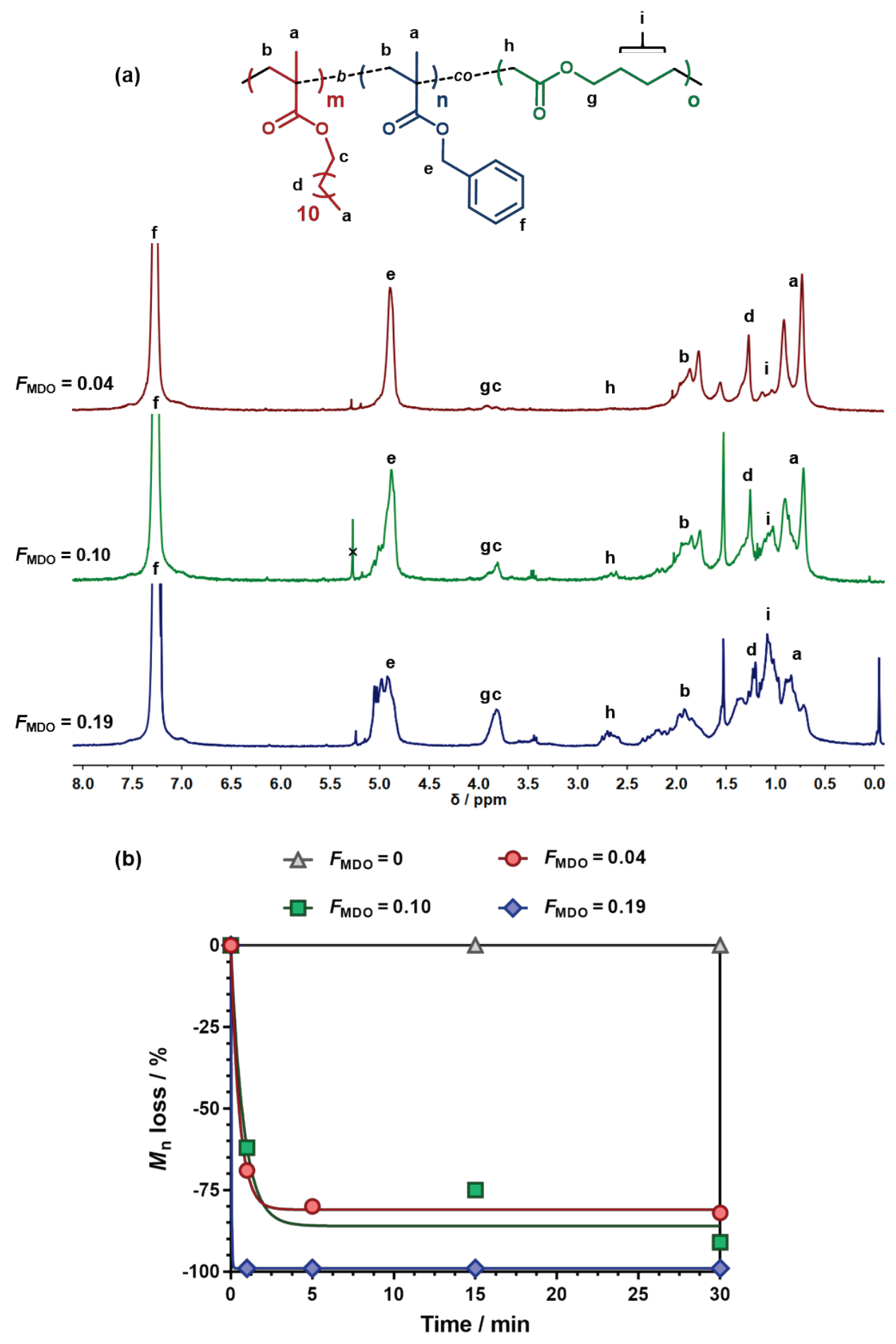

Figure 4. (a) ${ }^{1} \mathrm{H}$ NMR spectra in $\mathrm{CDCl}_{3}$ in the $0-8 \mathrm{ppm}$ region and (b) evolution with time of the percentage of number-average molar mass $\left(M_{\mathrm{n}}\right)$ loss during degradation under accelerated conditions (THF/MeOH, 2.5 wt. \% KOH) of $\mathbf{L}_{18}-\mathbf{B z} 150 \mathbf{M}$ copolymers as function of their molar fractions in $\mathrm{MDO}\left(F_{\mathrm{MDO}}\right)$. Plain lines for $F_{\mathrm{MDO}}=0.04,0.10$ and 0.19 represent a exponential one phase decay fit. 
L18-Bz150M nanoparticles were successfully formed for all values of $f_{\mathrm{MDO}, 0 \text {. They were }}$ characterized by DLS (Table S2) and exhibited an average intensity-diameter $\left(D_{\mathrm{z}}\right)$ of $102 \mathrm{~nm}$ $\left(F_{\mathrm{MDO}}=0.04\right), 140 \mathrm{~nm}\left(F_{\mathrm{MDO}}=0.10\right)$ and $202 \mathrm{~nm}\left(F_{\mathrm{MDO}}=0.19\right)$. Similarly to what has been observed for BMDO and MPDL, the higher $f_{\mathrm{MDO}, 0}$, the higher $D_{\mathrm{z}}$ and the higher the particle size distribution $(\mathrm{PSD}=0.18-0.34)$. The nanoparticles' colloidal properties determined by DLS were also in good agreement with TEM observations showing spheres with similar average

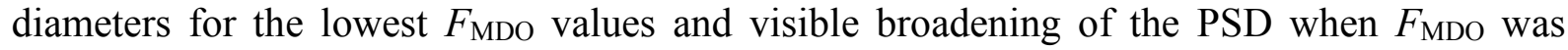
increased (Figure 5).
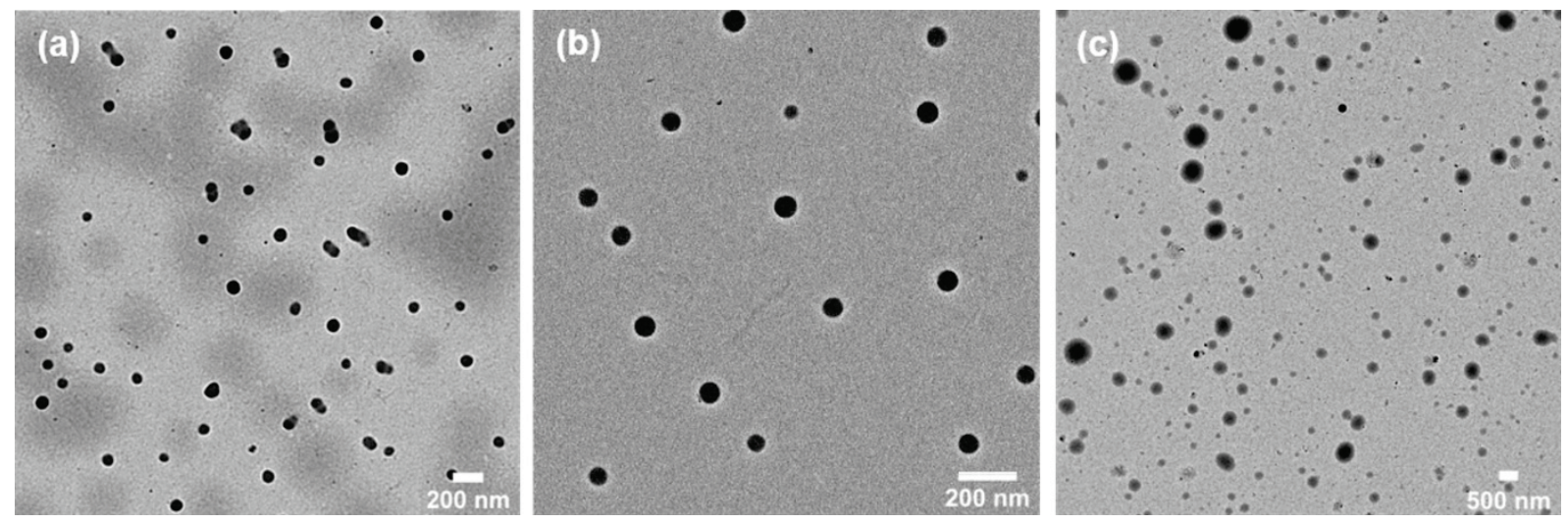

Figure 5. Representative TEM images of $\mathbf{L}_{18}-\mathbf{B z} \mathbf{z} \mathbf{1 5 0} \mathbf{M}$ nanoparticles: (a) $F_{\mathrm{MDO}}=0.04$; (b) $F_{\mathrm{MDO}}$ $=0.10 ;(\mathrm{c}) F_{\mathrm{MDO}}=0.19$.

It has previously been shown that inserting MPDL or BMDO units into the core block gave, at the end of the polymerization, spheres as the sole type of morphology whereas worms and especially vesicles were expected for such copolymer compositions. ${ }^{48}$ In fact, the same observation is made here for $\mathbf{L}_{\mathbf{1 8}}-\mathbf{B} \mathbf{z}_{150} \mathbf{M}$ nanoparticles ${ }^{31}$ as only spheres were obtained. Evolution of nanoparticles' morphology during the polymerization was also monitored at 
different time points for $f_{\mathrm{MDO}, 0}=0.2$, but showed spheres all the way, similarly to the same polymerization with MPDL (Figure S4). Conversely, when no CKA is added in the comonomer feed, a spheres/worms-to-vesicle transition is observed 6-8 h after the beginning of the polymerization (Table S3 and Figure S4). It has been shown that lowering the glass transition temperature $\left(T_{\mathrm{g}}\right)$ of the solvophobic block increased the mobility in the continuous phase and therefore enhanced the morphological transition of diblock copolymer nano-objects. ${ }^{49}$ Given $T_{\mathrm{g}, \mathrm{PMDO}} \sim-57{ }^{\circ} \mathrm{C}^{50}$ and $T_{\mathrm{g}, \mathrm{PMPDL}}=45 \pm 10{ }^{\circ} \mathrm{C},{ }^{45}$ a significant decrease of the $T_{\mathrm{g}}$ for MDOcontaining copolymers was therefore expected. DSC measurements performed on $\mathbf{L}_{\mathbf{1 8}}-\mathbf{B z} \mathbf{z} \mathbf{1 5 0}$, $\mathbf{L}_{18}-\mathbf{B z} 150 \mathbf{M}\left(F_{\mathrm{MDO}}=0.10\right.$. and 0.19$)$ and $\mathbf{L}_{18}-\mathbf{B z} \mathbf{z}_{150} \mathbf{M P}\left(F_{\mathrm{MPDL}}=0.25\right)$ copolymers confirmed this hypothesis (Figure S5) as: (i) inserting MDO into the copolymer decreased its $T_{\mathrm{g}}$ compared to that of the MDO-free copolymer $\left(T_{\mathrm{g}}=46.7^{\circ} \mathrm{C}\right.$ for $F_{\mathrm{MDO}}=0.10$ vs. $T_{\mathrm{g}}=52.0^{\circ} \mathrm{C}$ for $F_{\mathrm{MDO}}=$ 0 ); (ii) the more $\mathrm{MDO}$ in the copolymer, the lower its $T_{\mathrm{g}}$, as it reached $19.8^{\circ} \mathrm{C}$ for $F_{\mathrm{MDO}}=0.19$

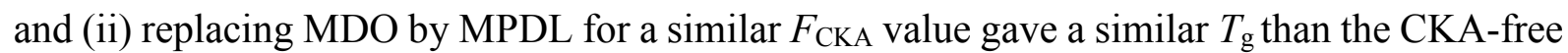
copolymer $\left(T_{\mathrm{g}}=56.8^{\circ} \mathrm{C}\right.$ for $\left.F_{\mathrm{MPDL}}=0.25\right)$. It was hoped that introducing MDO units into the solvophobic block would promote the formation of vesicles instead of spheres, but this hypothesis was eventually ruled out, which therefore calls for further investigations.

In conclusion, despite a less efficient control of the copolymerization than with MPDL or BMDO, MDO was similarly inserted into the copolymer backbone by rROPISA in heptane, leading to stable nanoparticles with PCL units in the core block and diblock copolymers that could be extensively degraded even for modest contents in MDO. 


\section{Surface-degradable nanoparticles by rROPISA}

Until now, tunable amounts of ester groups originated from different CKAs (MPDL, BMDO and MDO) were successfully inserted into the core of diblock copolymer nanoparticles via rROPISA. However, degradation of such copolymers left intact the solvophilic PLMA block. Hence, we wondered whether if it would be possible to engineer surface-degradable nanoparticles by rROPISA from CKA-containing solvophilic macro-CTAs.

Given the less efficient control of the copolymerization with MDO compared to that with MPDL and BMDO for the synthesis of core-degradable nanoparticles by rROPISA, first attempts to prepare degradable P(LMA-co-CKA) macro-CTAs were made by RAFT-mediated copolymerization between LMA and BMDO (or MPDL) at $f_{\mathrm{CKA}, 0}=0.4$ in anhydrous toluene using $\mathrm{CDB}$ as RAFT agent and AIBN as a radical initiator at $70{ }^{\circ} \mathrm{C}$ for $4 \mathrm{~h}$. Well-defined $\mathrm{P}\left(\mathrm{LMA}_{13}-\mathrm{co}-\mathrm{BMDO}_{0.7}\right)$ and $\mathrm{P}\left(\mathrm{LMA}_{17}-\mathrm{co}-\mathrm{MPDL}_{0.53}\right)$ copolymers were recovered after purification in methanol $\left(M_{\mathrm{n}, \mathrm{SEC}}=3600-4700 \mathrm{~g} \cdot \mathrm{mol}^{-1}, Ð=1.22-1.23\right.$, Table S4 and Figure S6). However, despite small but measurable amounts of CKA in the resultant copolymers $\left(F_{\mathrm{BMDO}}=0.05\right.$ and $\left.F_{\mathrm{MPDL}}=0.03\right)$, they did not show noticeable decrease in $M_{\mathrm{n}}$ upon degradation experiments under accelerated conditions (Figure S7), likely because of: (i) a high fraction of non-degradable, closed CKA units incorporated in the copolymer and/or (ii) too low $F_{\mathrm{CKA}}$ values given the chain lengths, resulting in most of the chains not having CKA units or having some mainly located close to the chain-end (because of unfavorable reactivity ratios ${ }^{36}$ ). Chain extension of $\mathrm{P}\left(\mathrm{LMA}_{17}-\mathrm{co}-\mathrm{MPDL}_{0.53}\right)$ was nevertheless performed by rROPISA in heptane at 90 ${ }^{\circ} \mathrm{C}$ and 15 wt.\% solids for $20 \mathrm{~h}$, which successfully conducted to the formation of stable, diblock copolymer nanoparticles of $126 \mathrm{~nm}$ in diameter (Table S5 and Figure S8). However, remaining 
traces of unreacted macro-CTA was observed in Figure S8b, similarly to what has already been obtained from CKA-free macro-CTAs. ${ }^{48}$ Interestingly, TEM images at different time points showed formation of vesicles with increasing membrane thickness with time, ultimately resulting in the loss of the vesicular morphology (Figure S9), similar to what has been observed with other PISA systems. ${ }^{12}$

A library of P(LMA-co-BMDO) macro-CTAs was then synthesized in order to find the best conditions to obtain degradable macro-CTAs without compromising the nanoparticles stability (we indeed envisioned that a too high amount of CKA in the solvophilic block would lead to colloidal instability). To promote insertion of open CKA units into the macro-CTA and therefore its degradation, the same polymerization conditions were employed except that $f_{\mathrm{BMDO}, 0}$ was varied from 0.2 to 0.7 and the reaction time was increased up to $20 \mathrm{~h}$ to reach higher

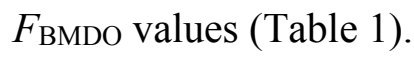

Table 1. Experimental Conditions and Macromolecular Characteristics of P(LMA-co-BMDO) Macro-CTA Synthesized at $70{ }^{\circ} \mathrm{C}$ for $20 \mathrm{~h}$ by RAFT-Mediated Solution Polymerization in Anhydrous Toluene.

\begin{tabular}{|c|c|c|c|c|c|c|c|}
\hline Copolymer $^{a}$ & $f_{\mathrm{CKA}, 0}$ & $F_{\mathrm{BMDO}}^{b}$ & $\begin{array}{c}\text { Opened } \\
\text { BMDO } \\
(\%)^{c}\end{array}$ & $\begin{array}{c}\text { LMA } \\
\text { Conv. } \\
(\%)^{d}\end{array}$ & $\begin{array}{c}M_{\mathrm{n}, \mathrm{NMR}} \\
\left(\mathrm{g} \cdot \mathrm{mol}^{-1}\right)^{e}\end{array}$ & $\begin{array}{c}M_{\mathrm{n}, \mathrm{SEC}} \\
\left(\mathrm{g} \cdot \mathrm{mol}^{-1}\right)^{f}\end{array}$ & $\boldsymbol{\theta}^{f}$ \\
\hline $\begin{array}{c}\text { P(LMA }{ }_{21}-c o- \\
\left.\text { BMDO }_{1.1}\right)\end{array}$ & 0.2 & 0.05 & 57 & 89 & 6200 & 5800 & 1.32 \\
\hline $\begin{array}{c}\text { P(LMA } \text { L1 }-c o- \\
\left.\text { BMDO }_{1.8}\right)\end{array}$ & 0.33 & 0.08 & 57 & 70 & 4600 & 5900 & 1.30 \\
\hline $\begin{array}{c}\text { P(LMA } \text { LM-co- }_{14} \\
\left.\text { BMDO }_{1.4}\right)\end{array}$ & 0.4 & 0.09 & 74 & 83 & 4600 & 4100 & 1.53 \\
\hline $\begin{array}{c}\text { P(LMA } \text { Li6-co- } \\
\left.\text { BMDO }_{1.7}\right)\end{array}$ & 0.5 & 0.10 & 77 & 69 & 3700 & 4500 & 1.37 \\
\hline
\end{tabular}




\begin{tabular}{cccccccc}
$\begin{array}{c}\text { P(LMA } \text { 17-co- }^{-} \\
\left.\text {BMDO }_{2.7}\right)\end{array}$ & 0.6 & 0.14 & 81 & 71 & 3900 & 4900 & 1.24 \\
$\begin{array}{c}\text { P(LMA } \text { (Lo- }^{-c o} \\
\left.\text { BMDO }_{3.9}\right)\end{array}$ & 0.7 & 0.24 & 76 & 59 & 4700 & 4200 & 1.32 \\
\hline
\end{tabular}

${ }^{a}$ Determined from $M_{\mathrm{n}, \mathrm{SEC}}$ and $F_{\mathrm{BMDO}} \cdot{ }^{b}$ Determined by ${ }^{1} \mathrm{H}$ NMR after precipitation. ${ }^{c}$ Determined by ${ }^{1} \mathrm{H}$ NMR by integrating the $2 \mathrm{H}$ of opened BMDO (5.0-5.2 ppm) and the $4 \mathrm{H}$ of closed BMDO (4.6-4.8 ppm). ${ }^{d}$ Determined by ${ }^{1} \mathrm{H}$ NMR. ${ }^{e}$ Determined by ${ }^{1} \mathrm{H}$ NMR by integrating the $2 \mathrm{H}$ of the RAFT agent (7.9-8.0 ppm), the $2 \mathrm{H}$ of BMDO (2.2-2.4 ppm) and the $2 \mathrm{H}$ of LMA (3.8-4.0 ppm). ${ }^{f}$ Determined SEC after precipitation.

$\mathrm{P}\left(\mathrm{LMA}-\mathrm{co}\right.$-BMDO) copolymers with $M_{\mathrm{n}, \mathrm{SEC}}=4 \quad 100-5900 \mathrm{~g} \cdot \mathrm{mol}^{-1}$ and rather narrow dispersity $(\bigoplus=1.24-1.53)$ were obtained and their structures were confirmed by ${ }^{1} \mathrm{H}$ NMR spectroscopy (Figure 6a). In particular, intensities of the peaks associated to BMDO protons (see protons $e, f$ and $h$ ) increased with an increase of $f_{\mathrm{BMDO}, 0}$, confirming the fine tuning of ester groups into the copolymer $\left(F_{\mathrm{BMDO}}=0.05-0.24\right)$, despite well-known discrepancies between $f_{\mathrm{BMDO}, 0}$ and $F_{\mathrm{BMDO}}$ because of unfavorable reactivity ratios between methacrylic esters and CKAs. $^{36}$

Their hydrolytic degradation under accelerated conditions was then performed and revealed minor degradation for copolymers with $F_{\mathrm{BMDO}}=0.05$ and 0.08 , and significant degradation when $F_{\mathrm{BMDO}} \geq 0.09$ (Figure $6 \mathrm{~b}$ and $\mathrm{S} 10$ ). Indeed, varying $F_{\mathrm{BMDO}}$ from 0.09 to 0.24 resulted in a progressive decrease of $M_{\mathrm{n}}$ after degradation from $-26 \%$ to $-83 \%$ (Figure $6 \mathrm{~b}$ and 6c), which is in good agreement between experimental $M_{\mathrm{n}}$ after degradation $\left(M_{\mathrm{n}, \exp }{ }^{\infty}\right)$ and theoretical ones $\left(M_{\mathrm{n}, \mathrm{th}^{\infty}}\right)$ (Table S6). 
(a)
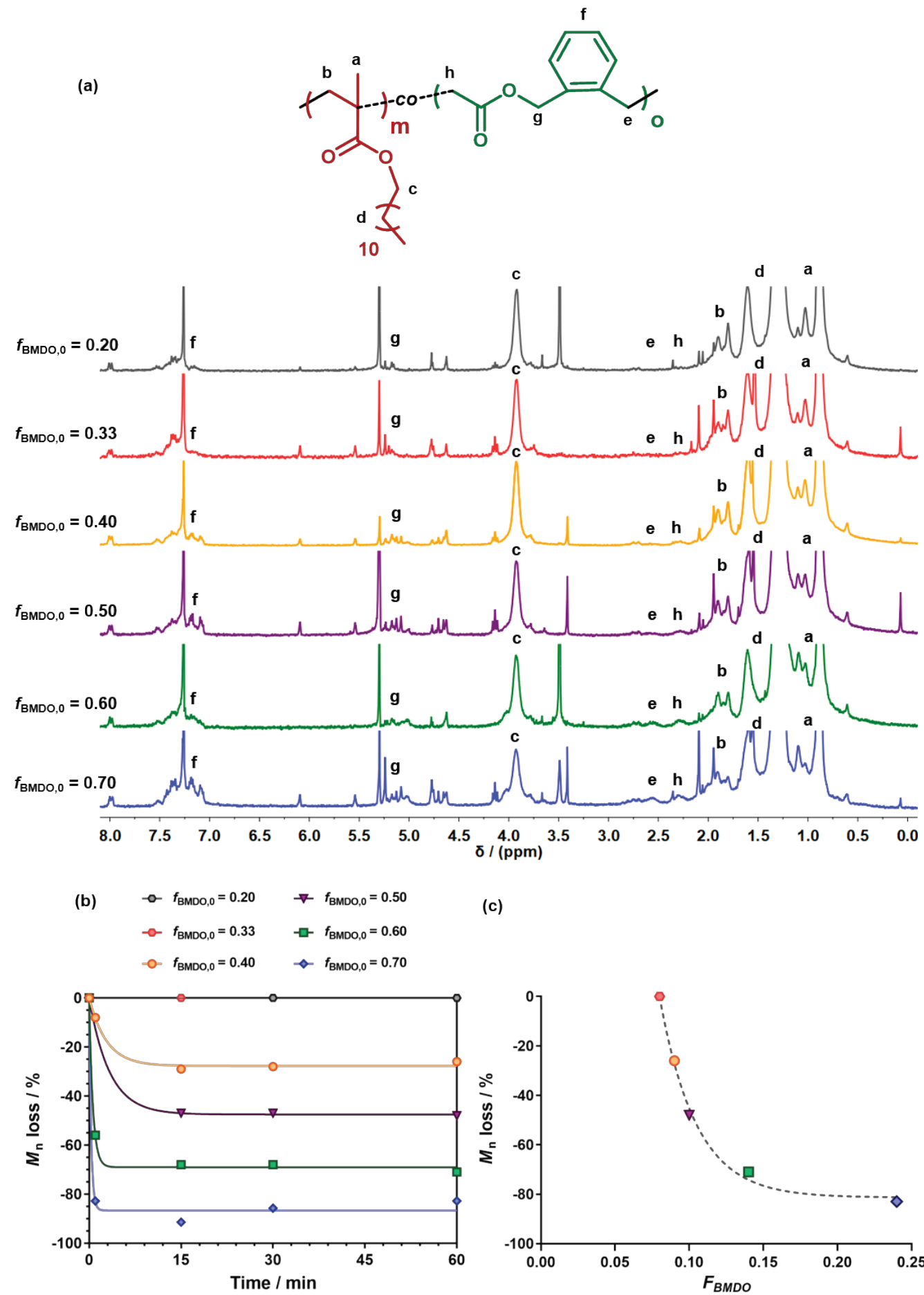

(c)

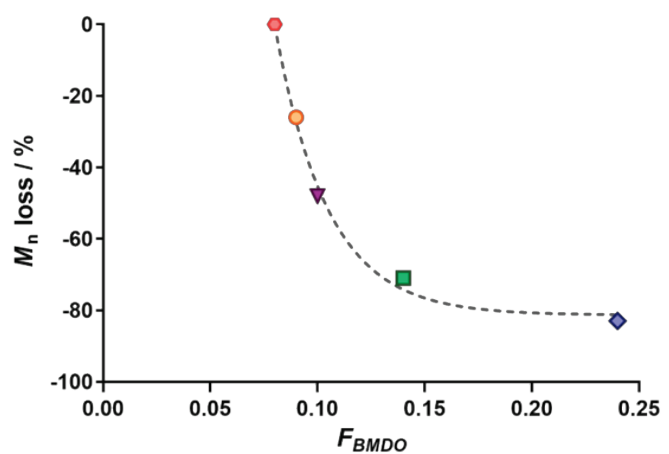

Figure 6. (a) ${ }^{1} \mathrm{H} \mathrm{NMR}$ spectra in $\mathrm{CDCl}_{3}$ in the $0-8$ ppm region of $\mathrm{P}(\mathrm{LMA}-\mathrm{co}-\mathrm{BMDO})$ macroCTAs as function of $f_{\mathrm{BMDO}, 0}$. (b) Evolution with time of the percentage of number-average molar mass $\left(M_{\mathrm{n}}\right)$ loss during degradation under accelerated conditions (THF/MeOH, $\left.2.5 \% \mathrm{KOH}\right)$ of P(LMA-co-BMDO) macro-CTA as function of $F_{B M D O}$ in the copolymer. Plain lines represent a exponential one phase decay fit. (c) Evolution of the $M_{\mathrm{n}}$ loss after degradation as function of $F_{\text {BMDO. }}$ 
rROPISA was then performed from PLMA 18 and from the previously synthetized P(LMA-coBMDO) macro-CTAs with BzMA (targeted $D P_{\mathrm{n}, \mathrm{BzMA}}=75$ ) in heptane at $90{ }^{\circ} \mathrm{C}$ for $8 \mathrm{~h}$ using T21s as a radical initiator (Table 2). rROPISA with PLMA 18 reached $78 \%$ conversion after $8 \mathrm{~h}$ and produced stable nanoparticles of well-defined PLMA- $b$-PBzMA diblock copolymers $\left(M_{\mathrm{n}, \mathrm{SEC}}=13000 \mathrm{~g} \cdot \mathrm{mol}^{-1}, Ð=1.09\right) . \mathrm{rROPISA}$ from P(LMA-co-BMDO) macro-CTAs reached $\sim 90 \%$ conversion after $8 \mathrm{~h}$ and rapidly led to formation of particles (e.g., after $\sim 30 \mathrm{~min}$ ) during the polymerization, which was faster than with PLMA 18 macro-CTA under the same conditions. The purified P(LMA-co-BMDO)- $b$-PBzMA copolymers exhibited higher $M_{\mathrm{n}}$ and dispersity $\left(M_{\mathrm{n}, \mathrm{SEC}}=90-115 \mathrm{~kg} \cdot \mathrm{mol}^{-1}, Ð=1.71-2.03\right)$ than without BMDO. This result can be explained by the lower livingness of $\mathrm{P}(\mathrm{LMA}-\mathrm{co}$-BMDO) macro-initiators, especially for those having the highest amounts of BMDO, as attested by the remaining amount of starting macro-CTA after chain-extension (Figure S11). However, stable P(LMA-co-BMDO)- $b$-PBzMA nanoparticles were obtained for $F_{\mathrm{BMDO}}$ values below 0.09 (Table 2), which indicated that a too high amount of BMDO units in the solvophilic block is detrimental regarding the colloidal stability of the nanoparticles. Indeed, the average diameter increased with $F_{\mathrm{BMDO}}$ from 68 to $848 \mathrm{~nm}$ until nanoparticles exhibited rapid colloidal instability (Table 2 and Figure S12). 
Table 2. Macromolecular and Colloidal Properties of P(LMA-co-BMDO)- $b$-PBzMA Diblock Copolymer Nanoparticles.

\begin{tabular}{|c|c|c|c|c|c|c|c|}
\hline Macro-CTA & $\begin{array}{c}\text { Conv. }(\%)^{a} / \\
\text { Time (h) }\end{array}$ & $\begin{array}{c}M_{\mathrm{n}, \mathrm{SEC}}{ }^{b} \\
\left({\left.\mathrm{~g} . \mathrm{mol}^{-1}\right)}^{-1}\right)\end{array}$ & $\begin{array}{c}M_{\mathrm{n}, \mathrm{NMR}} \\
\left(\mathrm{g} \cdot \mathrm{mol}^{-1}\right)^{c}\end{array}$ & $\boldsymbol{\oplus}^{b}$ & $\begin{array}{c}D_{\mathrm{z}}^{d} \\
(\mathrm{~nm})\end{array}$ & PSD $^{d}$ & Stability $^{e}$ \\
\hline PLMA18 & $78 / 8$ & 13000 & 16300 & 1.09 & 68 & 0.15 & yes \\
\hline P(LMA $\left.{ }_{21}-c o-B_{1.1}\right)$ & $93 / 8$ & 104400 & 145200 & 1.71 & 137 & 0.24 & yes \\
\hline P(LMA L $\left._{21}-c o-\mathrm{BMDO}_{1.8}\right)$ & $89 / 8$ & 90200 & 109400 & 1.78 & 181 & 0.26 & yes \\
\hline P(LMA14-co-BMDO1.4) & $93 / 8$ & 115400 & 140600 & 2.03 & 364 & 0.24 & yes \\
\hline P(LMA16-co-BMDO $\left.{ }_{1.7}\right)$ & $89 / 8$ & 97400 & 108400 & 1.88 & 848 & 0.20 & no \\
\hline P(LMA17-co-BMDO 2.7$)$ & n.d & n.d & n.d & n.d & n.d & n.d & no \\
\hline P(LMA12-co-BMDO3.9) & n.d & n.d & n.d & n.d & n.d & n.d & no \\
\hline
\end{tabular}

${ }^{a}$ BzMA conversion determined by ${ }^{1} \mathrm{H}$ NMR. ${ }^{b}$ Determined by SEC after precipitation of the copolymers.

${ }^{c}$ Determined by ${ }^{1} \mathrm{H}$ NMR by integrating the $2 \mathrm{H}$ of BzMA (4.9-5.1 ppm). ${ }^{d}$ Determined by DLS. ${ }^{e}$ Determined after $12 \mathrm{~h}$ by visual inspection to detect the presence of coagulum.

TEM images of $\mathbf{L}_{18}-\mathbf{B z}_{75}$ nanoparticles revealed the formation of spheres of low diameter (Figure 7a). In contrast, while nanoparticles with $F_{\mathrm{BMDO}}=0.05$ gave mainly spheres (Figure 7b), nanoporous spheres were obtained for copolymer nanoparticles with higher amounts of BMDO $\left(F_{\mathrm{BMDO}}=0.08\right.$ and 0.09$)$ in the solvophilic block (Figure $7 \mathrm{c}$ and $\left.7 \mathrm{~d}\right)$. Similar morphologies have already been observed, in particular by seeded RAFT dispersion and emulsion PISA. ${ }^{11,51,52}$ In our system, we might hypothesize that cavities come from early aggregation of particles which are less efficiently stabilized given the high fraction of BMDO at their surface, although a fusion-based mechanisms might also be envisionned. ${ }^{53}$ 

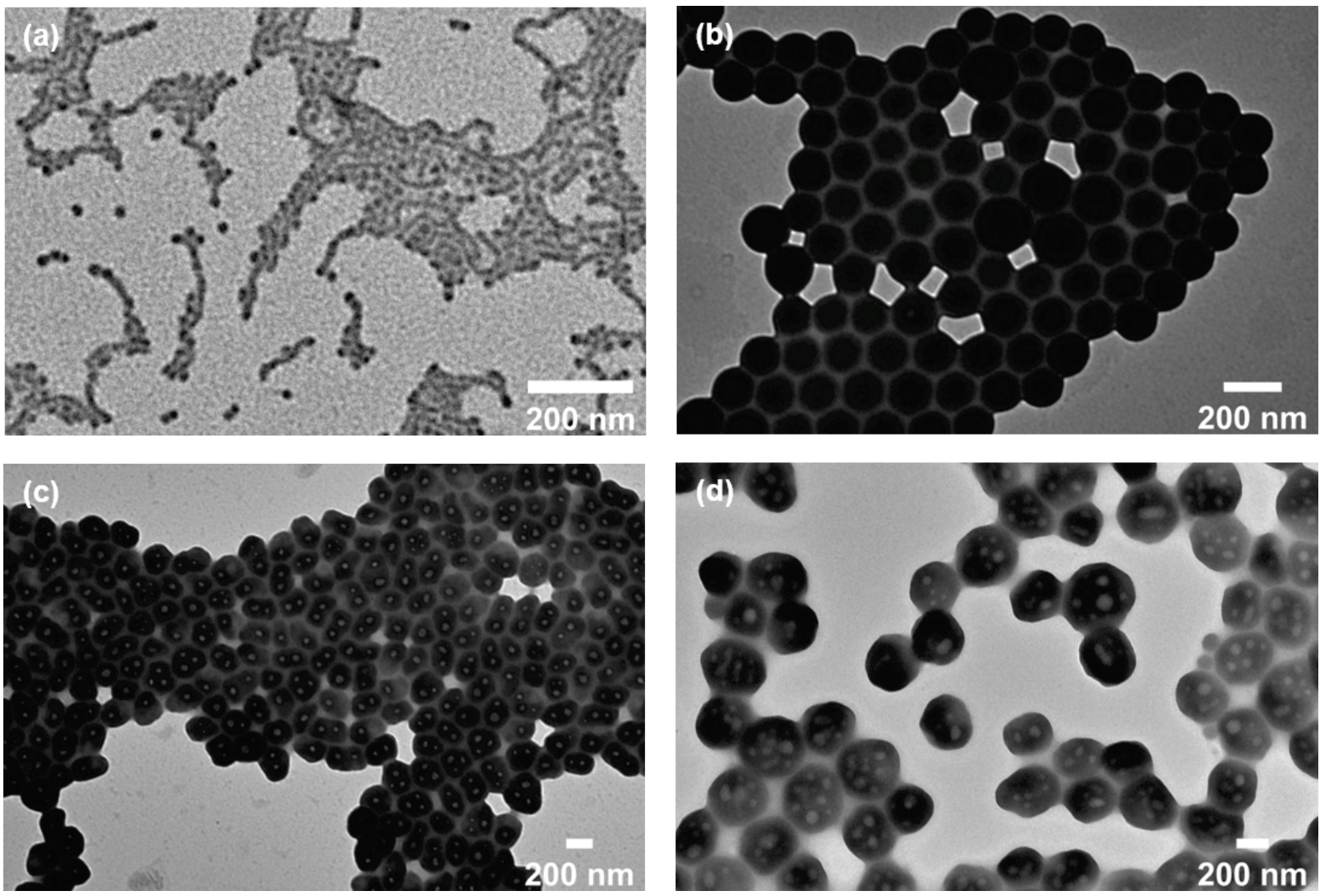

Figure 7. Representative TEM images of P(LMA-co-BMDO)- $b$-PBzMA nanoparticles, depending on the macro-CTA used: (a) $\mathrm{PLMA}_{18}$; (b) P(LMA 21 -co-BMDO 1.1$)$; (c) P(LMA 21 -co$\left.\mathrm{BMDO}_{1.8}\right)$; (d) $\mathrm{P}\left(\mathrm{LMA}_{14}-\mathrm{co}-\mathrm{BMDO}_{1.4}\right)$.

\section{Surface- and surface- plus core-degradable nanoparticles by rROPISA}

We also attempted to combine surface and core degradability to generate "all degradable" vinyl nanoparticles by rROPISA. By building upon our previous findings, we first prepared CKAcontaining macro-CTAs with higher $M_{\mathrm{n}}$ to improve the nanoparticle colloidal stability for high amounts of inserted CKA units in the solvophilic block. P(LMA $\left.28-c o-\mathrm{MPDL}_{2.1}\right)$ and $\mathrm{P}\left(\mathrm{LMA}_{20}{ }^{-}\right.$ co-BMDO 2.2 ) macro-CTAs were successfully synthesized by RAFT-mediated polymerization in anhydrous toluene using CDB as RAFT agent and AIBN as a radical initiator at $90{ }^{\circ} \mathrm{C}$ for 4 h (Table S7). We selected $90^{\circ} \mathrm{C}$ instead of $70^{\circ} \mathrm{C}$ as the polymerization temperature to promote a ring opening mechanism over the direct vinylic propagation. ${ }^{36}$ The macro-CTAs exhibited 
$M_{\mathrm{n}, \mathrm{SEC}}$ in the $5700-7600 \mathrm{~kg} \cdot \mathrm{mol}^{-1}$ range, low dispersities and relatively high fractions of CKA $\left(F_{\mathrm{MPDL}}=0.07\right.$ and $\left.F_{\mathrm{BMDO}}=0.10\right)$, giving a $M_{\mathrm{n}}$ loss of $\sim 40 \%$ after degradation (Figure S13). However, while degradation of $\mathrm{P}\left(\mathrm{LMA}_{20}-\mathrm{Co}-\mathrm{BMDO}_{2.2}\right)$ gave a clear shift of the SEC trace towards lower $M_{\mathrm{n}}$, only very small oligomers were produced for $\mathrm{P}\left(\mathrm{LMA}_{28}-c o-\mathrm{MPDL}_{2.1}\right)$ without noticeable shift of the whole peak, which may suggest that some chains do not have any MPDL units (given the lower fraction of MPDL) or MPDL units are more present close to the chain-end compared to BMDO units.

The macro-CTAs were then used to control the (rRO)PISA of BzMA in the presence or not of CKA (BMDO or MPDL, $f_{\mathrm{CKA}, 0}=0.4$ ) in heptane at $90{ }^{\circ} \mathrm{C}$ for $16 \mathrm{~h}$ using $\mathrm{T} 21 \mathrm{~s}$ as a radical initiator, to give four different suspensions of copolymers: two having CKAs only in the solvophilic block ( $\mathbf{L}_{20} \mathbf{B}_{2.2}-\mathbf{B z}_{75}$ and $\left.\mathbf{L}_{28} \mathbf{M P}_{2.1}-\mathbf{B z}_{150}\right)$ and two having CKAs in both blocks $\left(\mathbf{L}_{20} \mathbf{B}_{2.2}-\mathbf{B z}_{75} \mathbf{B}\right.$ and $\left.\mathbf{L}_{28} \mathbf{M P}_{2.1}-\mathbf{B z}_{150} \mathbf{M P}\right)$. Chain-extensions led to a rather good control of the polymerizations with $Ð \leq 1.60$ (after precipitation) and lower $M_{\mathrm{n}}$ (Table S8), despite noticeable fractions of non-living macro-CTAs (Figure S14). Also, stable nanoparticles were obtained in all cases with average diameters in the 61-166 nm range and relatively narrow PSD $(0.01-$ 0.25). ${ }^{1} \mathrm{H}$ NMR spectroscopy analysis of the purified "all-degradable" $\mathbf{L}_{20} \mathbf{B}_{2.2}-\mathbf{B z} 75 \mathbf{B}$ and $\mathbf{L}_{28} \mathbf{M P}_{2.1}-\mathbf{B z}_{150} \mathbf{M P}$ copolymers supported insertion of CKA during rROPISA, as total $F_{\mathrm{BMDO}}=$ 0.12 and total $F_{\mathrm{MPDL}}=0.15$, respectively (Figure $\mathrm{S} 15$ and $\left.\mathrm{S} 16\right)$.

Degradation under accelerated conditions of the four different copolymers agreed with the placement of CKA units within each copolymer. While copolymers bearing CKAs only in the solvophilic block gave slight/partial degradation (Figure $8 \mathrm{a}$ and $8 \mathrm{c}$ ), copolymers having CKA in both blocks gave significant degradation, as attested by the important shifts of the SEC traces 
towards low $M_{\mathrm{n}}$ values, resulting in $-95 \%$ (Figure $8 \mathrm{~b}$ ) and $-70 \%$ (Figure $8 \mathrm{~d}$ ) $M_{\mathrm{n}}$ loss. Interestingly, degradation of $\mathbf{L}_{\mathbf{2 0}} \mathbf{B}_{\mathbf{2 . 2}}-\mathbf{B} \mathbf{z} 75$ only produced very small oligomers without shifting the main SEC trace (Figure 8a), whereas degradation of $\mathbf{L}_{28} \mathbf{M} \mathbf{P}_{\mathbf{2 . 1}}-\mathbf{B} \mathbf{z}_{150}$ gave a slight shift of the SEC trace (Figure 8c) corresponding to a $M_{\mathrm{n}}$ loss of $7700 \mathrm{~g} \cdot \mathrm{mol}^{-1}$, which is almost exactly the $M_{\mathrm{n}}$ of the $\mathrm{P}\left(\mathrm{LMA}_{28}-\mathrm{co}-\mathrm{MPDL}_{2.1}\right)$ macro-CTA (Table S7). Even though drawing such conclusion from $M_{\mathrm{n}}$ values determined by conventional calibration must be taken with care, this observation seems to confirm the higher probability to find open MPDL units close to the solvophilic block's chain end compared to BMDO, which induced the release of nearly the entire solvophilic block upon degradation, conversely to release of very small oligomers with BMDO. 
(a)

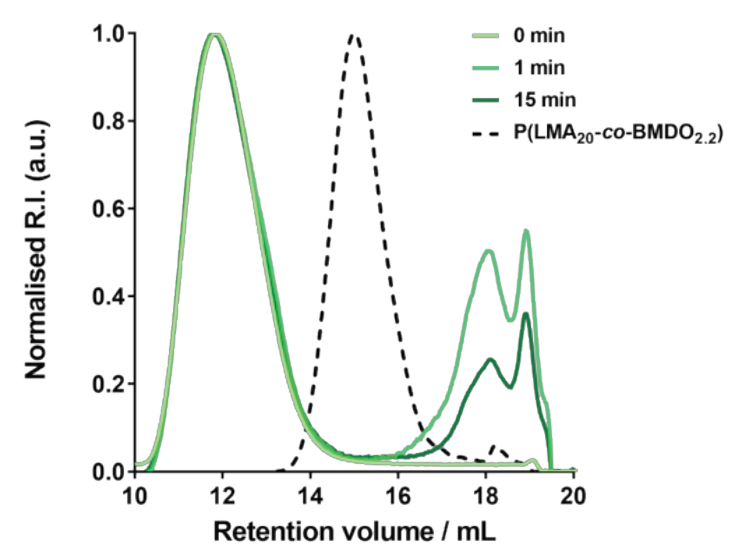

(c)

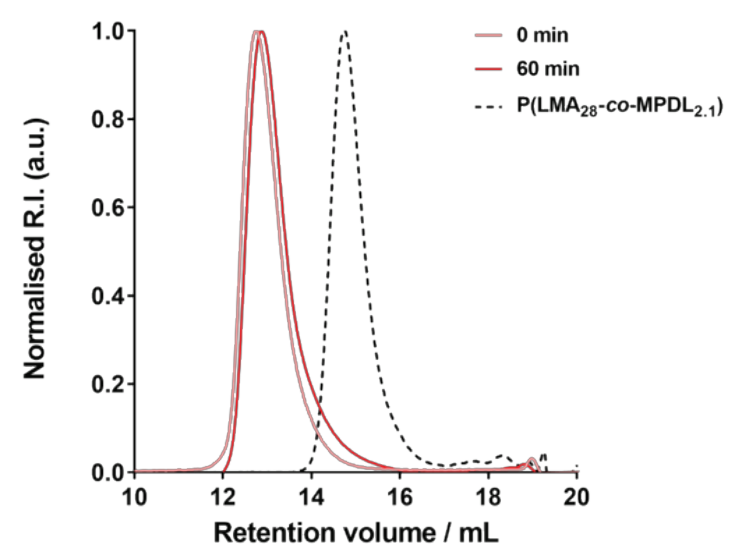

(b)

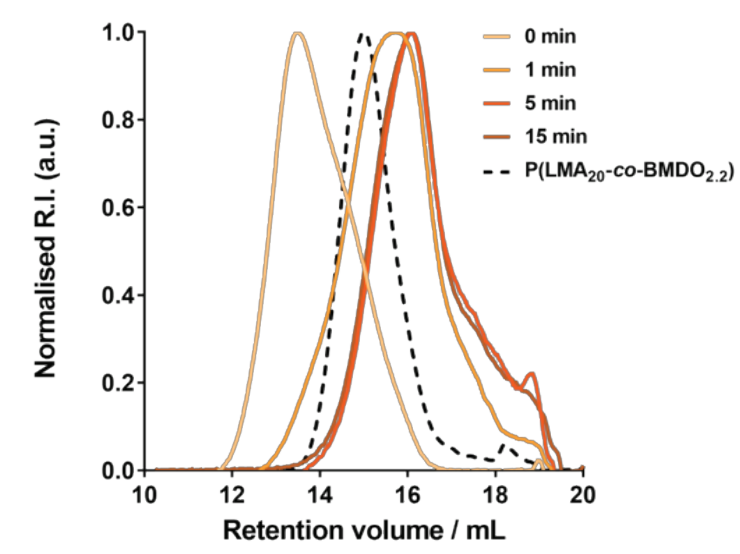

(d)

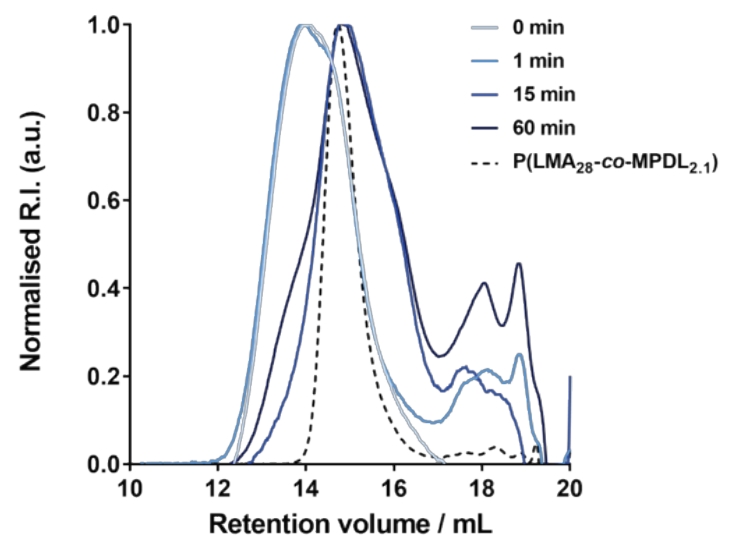

Figure 8. Evolution of the SEC chromatograms $\left(\mathrm{CHCl}_{3}\right.$ eluent with $0.1 \% \mathrm{v} / \mathrm{v}$ TFA $)$ at different time during the degradation under accelerated conditions (THF/MeOH, $\mathrm{KOH} 2.5 \%$ ) of: (a) $\mathbf{L}_{20} \mathbf{B}_{2.2-B z 75}$; (b) $\mathbf{L}_{20} \mathbf{B}_{2.2}-\mathbf{B z z}_{75} \mathbf{B}$ with $F_{\mathrm{BMDO}}=0.12$; (c) $\mathbf{L}_{28} \mathbf{M P}_{2.1}-\mathbf{B z}_{150}$ and (d) $\mathbf{L}_{28} \mathbf{M P}_{2.1}-$ Bz150MP with $F_{\mathrm{MPDL}}=0.15$.

\section{Conclusion}

In the present study, we first reported the successful application of RAFT-mediated rROPISA to the copolymerization between benzyl methacrylate (BzMA) and 2-methylene-1,3-dioxepane (MDO) from a PLMA macro-RAFT agent in heptane at $90{ }^{\circ} \mathrm{C}$, resulting in nanoparticles with tunable amounts of PCL-like ester groups $\left(F_{\mathrm{MDO}}=0.04-0.19\right)$ in the solvophobic block. Similarly to previous results with MPDL or BMDO, only spheres were obtained but a less 
efficient control of the polymerization was witnessed, which nevertheless led to very stable nanoparticles and successful degradation of the resulting copolymers under accelerated hydrolytic conditions.

CKA-containing PLMA macro-CTAs were then synthesized and used in rROPISA of BzMA to position ester groups at the surface of the nanoparticles. Even though livingness was only partial during chain extension, stable nanoparticles were obtained up to $F_{\mathrm{BMDO}}=0.09$ and exhibited unexpected porous morphology, which is unprecedented for CKA-containing polymers and represents an alternative way to reach such a morphology.

Finally, surface- plus core-degradable nanoparticles were obtained by performing rROPISA of MPDL or BMDO from CKA-containing macro-CTAs. However, optimization remains to be done in terms of livingness and control during the chain extension from CKAcontaining macro-CTA, and also in terms of colloidal stability for high CKA contents in the solvophilic block. It nonetheless represents a first step towards the synthesis of vinyl nanoparticles with site-specific degradability and towards "all degradable" nanoparticles in dispersed media.

\section{Acknowledgments}

We thank the China Scholarship Council (CSC) PhD fellowship (2017-2021) of CZ. The authors thank Claire Boulogne and Cynthia Gillet for technical assistance in TEM (I2BC, Gifsur-Yvette, France), and David Chapron and Sylviane Lesieur (IGPS, Univ Paris-Saclay) for their technical assistance in DSC. The CNRS is also acknowledged for financial support. 


\section{Graphical Abstract}

\section{rROP + PISA}

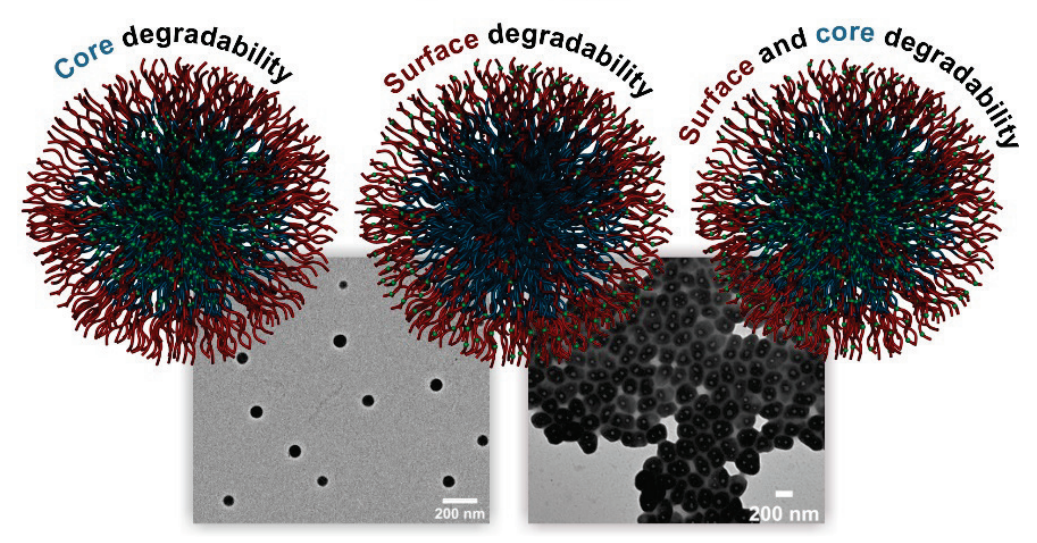

\section{References}

1. N. J. W. Penfold, J. Yeow, C. Boyer and S. P. Armes, ACS Macro Lett., 2019, 8, 1029-1054.

2. W. J. Zhang, C. Y. Hong and C. Y. Pan, Macromol. Rapid Commun., 2019, 40, e1800279.

3. N. J. Warren and S. P. Armes, J. Am. Chem. Soc., 2014, 136, 10174-10185.

4. X. Wang and Z. An, Macromol. Rapid Commun., 2019, 40, e1800325.

5. S. L. Canning, G. N. Smith and S. P. Armes, Macromolecules, 2016, 49, 1985-2001.

6. B. Charleux, G. Delaittre, J. Rieger and F. D'Agosto, Macromolecules, 2012, 45, 6753-6765.

7. D. Zehm, L. P. D. Ratcliffe and S. P. Armes, Macromolecules, 2013, 46, 128-139.

8. P. Gurnani, C. P. Bray, R. A. E. Richardson, R. Peltier and S. Perrier, Macromol. Rapid Commun., 2019, 40, 1800314.

9. C. A. Figg, R. N. Carmean, K. C. Bentz, S. Mukherjee, D. A. Savin and B. S. Sumerlin, Macromolecules, 2017, 50, 935-943.

10. N. J. W. Penfold, J. R. Whatley and S. P. Armes, Macromolecules, 2019, 52, 1653-1662.

11. M. Huo, M. Zeng, D. Wu, Y. Wei and J. Yuan, Polym. Chem., 2018, 9, 912-919.

12. N. J. Warren, O. O. Mykhaylyk, A. J. Ryan, M. Williams, T. Doussineau, P. Dugourd, R. Antoine, G. Portale and S. P. Armes, J. Am. Chem. Soc., 2015, 137, 1929-1937.

13. C. Gonzato, M. Semsarilar, E. R. Jones, F. Li, G. J. P. Krooshof, P. Wyman, O. O. Mykhaylyk, R. Tuinier and S. P. Armes, J. Am. Chem. Soc., 2014, 136, 11100-11106.

14. W.-J. Zhang, C.-Y. Hong and C.-Y. Pan, Macromolecules, 2014, 47, 1664-1671.

15. C. Bergerbit, F. Baffie, A. Wolpers, P.-Y. Dugas, O. Boyron, M. Taam, M. Lansalot, V. Monteil and F. D'Agosto, Angewandte Chemie International Edition, 2020, 59, 10385-10390. 
16. R. G. Gilbert, Emulsion Polymerization: A Mechanistic Approach, Academic Press, London, 1995.

17. D. Le, M. Dilger, V. Pertici, S. Diabaté, D. Gigmes, C. Weiss and G. Delaittre, Angewandte Chemie International Edition, 2019, 58, 4725-4731.

18. G. Wang, M. Schmitt, Z. Wang, B. Lee, X. Pan, L. Fu, J. Yan, S. Li, G. Xie, M. R. Bockstaller and K. Matyjaszewski, Macromolecules, 2016, 49, 8605-8615.

19. D. B. Wright, M. A. Touve, L. Adamiak and N. C. Gianneschi, ACS Macro Lett., 2017, 6, $925-$ 929.

20. J. Yeow, J. Xu and C. Boyer, ACS Macro Lett., 2015, 4, 984-990.

21. J. Yeow, O. R. Sugita and C. Boyer, ACS Macro Lett., 2016, 5, 558-564.

22. M. J. Derry, L. A. Fielding and S. P. Armes, Prog. Polym. Sci., 2016, 52, 1-18.

23. X. Wang, S. Man, J. Zheng and Z. An, ACS Macro Lett., 2018, 7, 1461-1467.

24. J. Engström, T. Benselfelt, L. Wågberg, F. D'Agosto, M. Lansalot, A. Carlmark and E. Malmström, Nanoscale, 2019, 11, 4287-4302.

25. X. Zhang, A. F. Cardozo, S. Chen, W. Zhang, C. Julcour, M. Lansalot, J. F. Blanco, F. Gayet, H. Delmas, B. Charleux, E. Manoury, F. D'Agosto and R. Poli, Chemistry, 2014, 20, 1550515517.

26. S. Kaga, N. P. Truong, L. Esser, D. Senyschyn, A. Sanyal, R. Sanyal, J. F. Quinn, T. P. Davis, L. M. Kaminskas and M. R. Whittaker, Biomacromolecules, 2017, 18, 3963-3970.

27. S. Varlas, J. C. Foster, P. G. Georgiou, R. Keogh, J. T. Husband, D. S. Williams and R. K. O'Reilly, Nanoscale, 2019, 11, 12643-12654.

28. L. D. Blackman, S. Varlas, M. C. Arno, A. Fayter, M. I. Gibson and R. K. O'Reilly, ACS Macro Lett., 2017, 6, 1263-1267.

29. V. Ladmiral, M. Semsarilar, I. Canton and S. P. Armes, J. Am. Chem. Soc., 2013, 135, 1357413581.

30. A. Musyanovych, J. Schmitz-Wienke, V. Mailänder, P. Walther and K. Landfester, Macromol. Biosci., 2008, 8, 127-139.

31. E. Guégain, C. Zhu, E. Giovanardi and J. Nicolas, Macromolecules, 2019, 52, 3612-3624.

32. P. J. Hurst, A. M. Rakowski and J. P. Patterson, Nature Commun., 2020, 11, 4690.

33. J. Jiang, X. Zhang, Z. Fan and J. Du, ACS Macro Lett., 2019, 8, 1216-1221.

34. V. Delplace and J. Nicolas, Nature Chem., 2015, 7, 771-784.

35. S. Agarwal, Polym. Chem., 2010, 1, 953-964.

36. A. Tardy, J. Nicolas, D. Gigmes, C. Lefay and Y. Guillaneuf, Chem. Rev., 2017, 117, 13191406.

37. A. W. Jackson, Polym. Chem., 2020, 11, 3525-3545. 
38. A. Tardy, N. Gil, C. M. Plummer, D. Siri, D. Gigmes, C. Lefay and Y. Guillaneuf, Angew. Chem., Int. Ed., 2020, 59, 14517-14526.

39. J.-B. Lena, A. W. Jackson, L. R. Chennamaneni, C. T. Wong, F. Lim, Y. Andriani, P. Thoniyot and A. M. Van Herk, Macromolecules, 2020, 53, 3994-4011.

40. A. Tardy, J. C. Honoré, J. Tran, D. Siri, V. Delplace, I. Bataille, D. Letourneur, J. Perrier, C. Nicoletti, M. Maresca, C. Lefay, D. Gigmes, J. Nicolas and Y. Guillaneuf, Angew. Chem., Int. Ed., 2017, 56, 16515-16520.

41. J. Folini, C.-H. Huang, J. C. Anderson, W. P. Meier and J. Gaitzsch, Polym. Chem., 2019, 10, 5285-5288.

42. J. Tran, T. Pesenti, J. Cressonnier, C. Lefay, D. Gigmes, Y. Guillaneuf and J. Nicolas, Biomacromolecules, 2019, 20, 305-317.

43. J. Liu, J. Wang, Y.-f. Xue, T.-t. Chen, D.-n. Huang, Y.-x. Wang, K.-f. Ren, Y.-b. Wang, G.-s. Fu and J. Ji, J. Mater. Chem. B, 2020, 8, 5361-5368.

44. T. Pesenti and J. Nicolas, ACS Macro Lett., 2020, in press.

45. J. Tran, E. Guegain, N. Ibrahim, S. Harrisson and J. Nicolas, Polym. Chem., 2016, 7, 44274435 .

46. G. G. Hedir, C. A. Bell, R. K. O'Reilly and A. P. Dove, Biomacromolecules, 2015, 16, 20492058 .

47. C. A. Bell, G. G. Hedir, R. K. O'Reilly and A. P. Dove, Polym. Chem., 2015, 6, 7447-7454.

48. L. A. Fielding, M. J. Derry, V. Ladmiral, J. Rosselgong, A. M. Rodrigues, L. P. D. Ratcliffe, S. Sugihara and S. P. Armes, Chem. Sci., 2013, 4, 2081-2087.

49. J. Zhou, W. Zhang, C. Hong and C. Pan, Polym. Chem., 2016, 7, 3259-3267.

50. S. Jin and K. E. Gonsalves, Macromolecules, 1997, 30, 3104-3106.

51. H. Zhou, C. Liu, Y. Qu, C. Gao, K. Shi and W. Zhang, Macromolecules, 2016, 49, 8167-8176.

52. C. Gao, J. Wu, H. Zhou, Y. Qu, B. Li and W. Zhang, Macromolecules, 2016, 49, 4490-4500.

53. L. R. Parent, E. Bakalis, A. Ramírez-Hernández, J. K. Kammeyer, C. Park, J. de Pablo, F. Zerbetto, J. P. Patterson and N. C. Gianneschi, J. Am. Chem. Soc., 2017, 139, 17140-17151. 